\title{
Conformational analysis of cycloalkanes
}

\author{
Veljko Dragojlovic ${ }^{1}$
}

Received: 16 April 2015/Accepted: 18 July 2015/Published online: 12 August 2015

(C) Springer International Publishing 2015

\begin{abstract}
Conformational analysis is a comparatively new area of organic chemistry that has been developed well after the theories of organic reactions, bonding in organic compounds and stereochemistry. It was only in the second half of twentieth century that its importance was fully recognized and its central role with respect to bonding, reactivity and stability of organic compounds was appreciated. Fundamental concepts of conformational analysis, a deeper discussion of the conformational analysis of small and common rings and an introduction into the conformational analysis of medium and large rings are presented at a level suitable for an introductory organic chemistry course. This discussion aims to provide a better understanding of the complex relationship among different types of strain, while also discussing the factors that determine stability of a particular conformation. Finally, the unique nature of each class of cycloalkanes is explored.
\end{abstract}

Keywords Physical organic chemistry - Conformational analysis - Strain - Cycloalkanes - Medium rings ·

Large rings

\section{Introduction}

\section{Conformational analysis}

In 1874, Van't Hoff and Le Bell independently proposed that the four valencies of carbon are directed toward the corners of a tetrahedron with the carbon atom at its center

Veljko Dragojlovic

vdragojl@fau.edu

1 Wilkes Honors College of Florida Atlantic University, 5353 Parkside Drive, Jupiter, FL 33458, USA
$[1,2]$. The model was adopted by other chemists, applied to more complex molecules and further developed culminating with Barton's 1950 paper in which he set foundations of modern conformational analysis [3].

Configuration of a molecule denotes three-dimensional arrangement of atoms in space. The infinite number of different arrangements of atoms in space that is a result of rotation about a single bond is called conformational isomers, conformers or conformations. Yet another less common term is rotamers.

It is often said that a rotation about a single bond is "free". The statement is not meant to imply that there is no energy barrier to a rotation, but that rotation is spontaneous at room temperature. Thus, conformations are not actual isomers as they cannot be separated and isolated. They are different shapes of the same molecule. Conformational analysis is the study of kinetic and thermodynamic properties of molecules that are conformation dependent such as the existence of a preferred conformation, energies and populations of different conformational arrangements, and chemical consequences of it.

\section{Strain in acyclic compounds}

Before one can carry out conformational analysis of cyclic compounds, it is necessary to introduce fundamental concepts on examples of simple acyclic compounds. Furthermore, stability of a cyclic compound is sometimes estimated or calculated by comparing its energy to the estimated energy of the corresponding acyclic equivalent.

\section{Conformational analysis of ethane}

One of the simplest organic compounds that exists in different conformations is ethane. Different conformations of 
<smiles>[CH2][CH2+]</smiles><smiles>CC</smiles>

a<smiles>C1CCC2C(C1)CC1CCC12</smiles><smiles>C1CCC2CCCCC2C1</smiles>

b<smiles>CC</smiles>

Fig. 1 Formulas of ethane. In each case, staggered conformation is on the left and eclipsing conformation is on the right. a Perspective formulas. b Newman Projections. c Sawhorse formulas

ethane are a result of rotation about the carbon-carbon bond. Obviously, there is an infinite number of such conformations. Fortunately, to carry out conformational analysis of ethane one needs to consider only the two extreme conformations-staggered and eclipsed. In the eclipsed conformation, each of the carbon-hydrogen bonds is in the same plane as the carbon-hydrogen bonds on the neighboring carbon. In a staggered conformation, each of the carbon-hydrogen bonds is in between the two carbon-hydrogen bonds on the neighboring carbon atom. Configuration of an organic compound is usually represented by a perspective formula. For the purpose of conformational analysis, the Newman projection and the sawhorse formula are frequently more suitable.

In a perspective formula of ethane, two carbons and two hydrogens (one bonded to each carbon) are in the plane of the drawing. Two hydrogens (again bonded to each carbon) are above the plane of the drawing, project towards the viewer, and are represented by wedge bonds. The remaining two hydrogen atoms are behind the plane of the

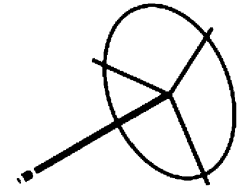

a

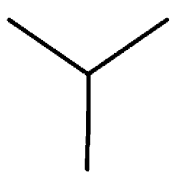

b

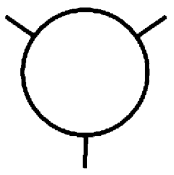

C

Fig. 2 a Newman's formulas were inspired by an umbrella. b The valence bonds to carbon 1 are viewed as one views the spokes of an umbrella when the eye is at the handle. $\mathbf{c}$ The valence bonds to carbon 2 are viewed as though the eye were at the tip

Fig. 3 Dihedral angle a represented by a sawhorse projection $\mathbf{b}$ represented by a Newman projection $\mathbf{c}$ of eclipsing and staggered conformations of ethane drawing, project away from the viewer, and are represented by dash bonds (Fig. 1a).

In a Newman projection formula one observes the molecule along the bond joining the two atoms, in this case the carbon-carbon bond of ethane (Fig. 1b). As a convention, the carbon nearest to the eye is labeled as carbon 1 and farthest from the eye carbon 2 . The closer carbon atom is represented as a dot and the remaining three substituents are represented as attached to it with the angles between the substituents of $120^{\circ}$. The back carbon is represented as a circle and its substituents are shown as appearing over the horizon of that atom. If the substituents eclipse each other as in the case of ethene's hydrogens on the right, we "cheat" and rotate the substituents a bit so that we can represent all of them. Newman described his formulas as being inspired by an umbrella (Fig. 2a): "the valence bonds to carbon 1 are viewed as one views the spokes of an umbrella when the eye is at the handle (Fig. 2b), and the valence bonds to carbon $\mathbf{2}$ are viewed as though the eye were at the tip (Fig. 2c)" [4].

Similar to the Newman formula, a sawhorse formula is useful if one wants to represent the configuration of the two neighboring atoms (Fig. 1c). One draws an elongated bond connecting these two atoms and, again similar to the Newman projection formula, the substituents on each carbon atom are represented as if the angle between them was $120^{\circ}$ _resembling a letter $\mathrm{Y}$ or an inverted letter Y.

Dihedral angle (also called torsion angle), $\theta$, is the angle between two planes. Each plane is defined by three atoms $\mathrm{C}-\mathrm{C}-\mathrm{X}$ and $\mathrm{C}-\mathrm{C}-\mathrm{Y}$ as shown below (Fig. 3a). Therefore, it is the angle made by the bonds on the adjacent atoms. Newman projection is particularly convenient for observing dihedral angle as it is the angle formed by the $\mathrm{C}-\mathrm{X}$ and

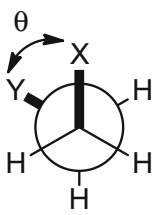

b
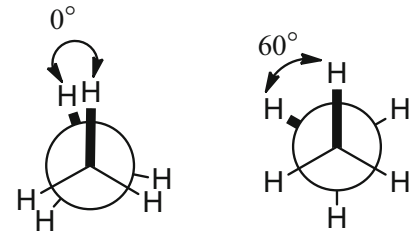

C 
$\mathrm{C}-\mathrm{Y}$ bonds (Fig. 3b). In the case of ethane, the dihedral angle is the angle between the carbon-hydrogen bonds on the two carbon atoms (Fig. 3c).

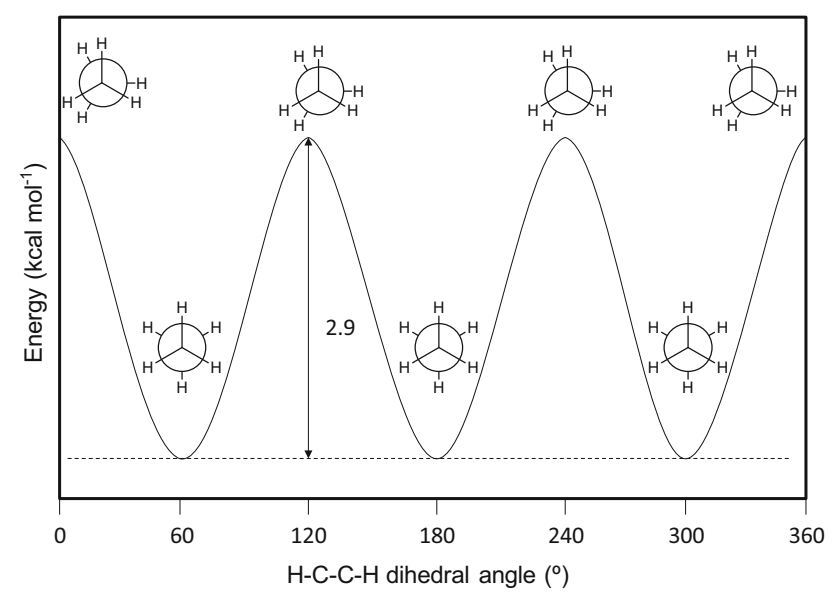

Fig. 4 Energy diagram for rotation about the carbon-carbon bond in ethane<smiles>O=CC1CCCCCO1</smiles>

a

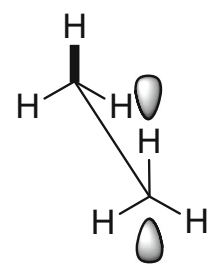

b
Fig. 5 a Delocalization of electrons from the filled bonding $\mathrm{C}-\mathrm{H}$ orbital (bold) into the antibonding $\mathrm{C}-\mathrm{H}^{*}$ orbital (one of the six interactions shown). b Hyperconjugation is not possible in an eclipsed conformation
A rotation about a carbon-carbon bond by $60^{\circ}$ converts an eclipsed conformation into a staggered one and vice versa. Dihedral angles of $0^{\circ}, 120^{\circ}$ and $240^{\circ}$ represent eclipsed conformations, whereas dihedral angles of $60^{\circ}$, $180^{\circ}$ and $300^{\circ}$ represent staggered conformations. An eclipsed conformation is higher in energy compared to a staggered conformation (Fig. 4). The change in energy due to the rotation from a staggered to an eclipsed conformation is called torsional strain (a less common name is rotational strain). Conformations intermediate between staggered and eclipsed are intermediate in energy and are called skew conformations. Skew conformations are important when considering conformations of cyclic compounds.

The reason for the energy difference is due to stabilization of the staggered conformation by hyperconjugation [5]. In ethane, hyperconjugation involves delocalization of electrons from the filled bonding $\mathrm{C}-\mathrm{H}$ orbital (shown in bold), which acts as a donor, into the vacant antibonding $\mathrm{C}-\mathrm{H}^{*}$ orbital, which acts as an acceptor (Fig. 5a). Such electron delocalization between bonding and antibonding $\mathrm{C}-\mathrm{H}$ orbitals is not possible in an eclipsed conformation (Fig. 5b).

\section{Conformational analysis of propane}

Conformational properties of propane are very similar to that of ethane. The two extreme conformations correspond to dihedral angles of $0^{\circ}, 120^{\circ}$ and $240^{\circ}$ (eclipsed) and of $60^{\circ}, 180^{\circ}$ and $300^{\circ}$ (staggered) (Fig. 6). A plot of energy versus dihedral angle is similar to that of ethane, except that the rotational barrier is slightly higher (Fig. 7). Besides the torsional strain, the barrier for rotation now involves an additional strain due to the eclipsing of a methyl group with a hydrogen atom. Such strain is called steric strain, or steric<smiles>[CH][CH][CH]</smiles><smiles>[CH][CH][CH]</smiles>

a<smiles>CC1CC2CC3CC2CC13</smiles><smiles>CC1CCCCC1</smiles>

b<smiles>CCC</smiles><smiles>CCC</smiles>

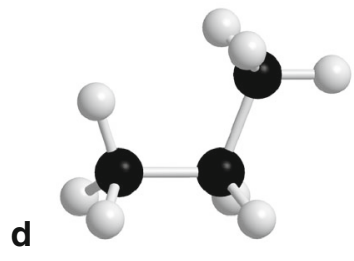

\section{d}

Fig. 6 Representations of propane with a staggered conformation on the left and an eclipsing on the right: a Perspective formula. b Newman projection. c Sawhorse formula. d In the eclipsing

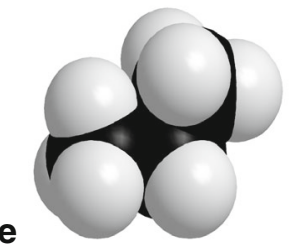

conformation of propane $\mathrm{C}-\mathrm{CH}_{3}$ bond is eclipsing $\mathrm{C}-\mathrm{H}$ bond. e The resulting steric strain is best seen in a space-filling model 
hindrance (Fig. 6e). In case of propane, steric strain increases energy of the rotational barrier by additional $0.5 \mathrm{kcal} \mathrm{mol}^{-1}$.

Steric strain in propane is actually more serious than it may appear from a simple analysis of the two extreme conformations. Two geminal methyl groups raise not only the energy of the eclipsed but also of the staggered conformation. Therefore, while the energy difference between the staggered and the eclipsed conformation of propane is only $0.5 \mathrm{kcal} \mathrm{mol}^{-1}$ higher compared to ethane, one must keep in mind that steric hindrance also raises the energy of the staggered conformation. To relieve steric strain, the $\mathrm{C}-$ $\mathrm{C}-\mathrm{C}$ angle in propane increases from the ideal tetrahedral angle of $109.5^{\circ}$ to $111.7^{\circ}$ (Fig. 8). Since in a cycloalkane each carbon atom is bonded to two other carbons, propane bond angle is used as a "standard" to which bond angles of cycloalkanes are compared.

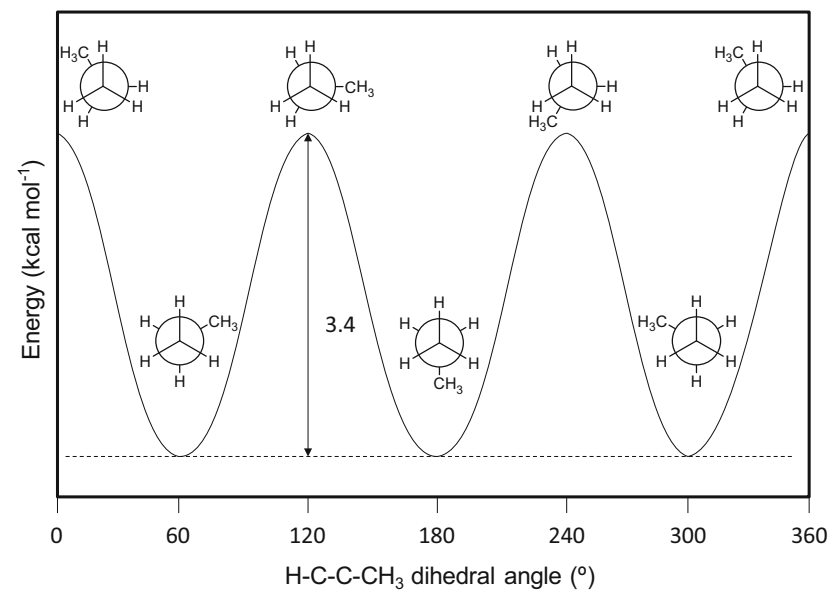

Fig. 7 Energy diagram for rotation about the carbon-carbon bond in propane

Fig. 8 In the staggered conformation of propane some steric strain is present, which forces $\mathrm{C}-\mathrm{C}-\mathrm{C}$ bond to adopt $111.7^{\circ}$ bond angle

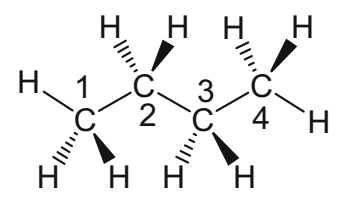

a

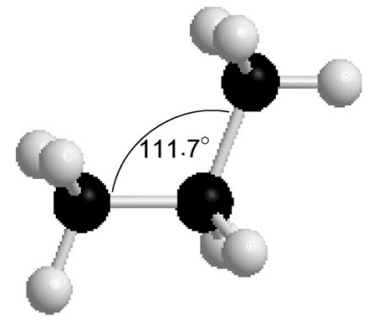<smiles>CC1C2CC3CC1C(C)C3C2</smiles>

b<smiles>CCC(C)C</smiles>

c
Fig. 9 Representations of butane: a perspective formula. b Newman projection. c Sawhorse formula

\section{Conformational analysis of butane}

\section{Representations of butane}

A perspective drawing of a staggered conformation of butane has all the carbon atoms as well as the two hydrogen atoms at each end of the chain (terminal hydrogen atoms) in a single plane - the plane of the drawing. That leaves two hydrogen atoms on each carbon atom. One of the hydrogen atoms is above the plane of the drawing (wedge bond) and the other one is below the plane (dash bond) (Fig. 9a). In a Newman projection (Fig. 9b) and a sawhorse formula (Fig. 9c) of butane the two terminal methyl groups (carbons 1 and 4) are represented as substituents.

A molecule of ethane has only one type of each staggered and eclipsed conformation. In a more complex molecule, such as butane, one can identify different types of staggered and eclipsed conformations.

\section{Gauche and anti conformers of butane}

According to IUPAC nomenclature (Fig. 10): "Stereochemical arrangements corresponding to torsion angles between $0^{\circ}$ and $\pm 90^{\circ}$ are called syn $(s)$, those corresponding to torsion angles between $\pm 90^{\circ}$ and $180^{\circ}$ anti $(a)$. Similarly, arrangements corresponding to torsion angles between $30^{\circ}$ and $150^{\circ}$ or between $-30^{\circ}$ and $-150^{\circ}$ are called clinal $(c)$ and those between $0^{\circ}$ and $\pm 30^{\circ}$ or $\pm 150^{\circ}$ and $180^{\circ}$ are called periplanar $(p)$. The two types of terms can be combined so as to define four ranges of torsion angle; $0^{\circ}$ to $\pm 30^{\circ}$ synperiplanar $(s p) ; 30^{\circ}$ to $90^{\circ}$ and $-30^{\circ}$ to $-90^{\circ}$ synclinal $(s c) ; 90^{\circ}$ to $150^{\circ}$, and $-90^{\circ}$ to $-150^{\circ}$ anticlinal $(a c) ; \pm 150^{\circ}$ to $180^{\circ}$ antiperiplanar (ap). The synperiplanar conformation is also known as the syn- or cis-conformation; antiperiplanar as anti or trans and synclinal as gauche or skew" [6].

A dihedral angle in butane can be defined as the angle between $\mathrm{C}^{1}-\mathrm{C}^{2}$ and $\mathrm{C}^{3}-\mathrm{C}^{4}$ bonds. Rotation about the $\mathrm{C}-\mathrm{C}$ bond in $60^{\circ}$ increments results in molecule of butane passing through a sequence of syn, gauche, anticlinal, anti,

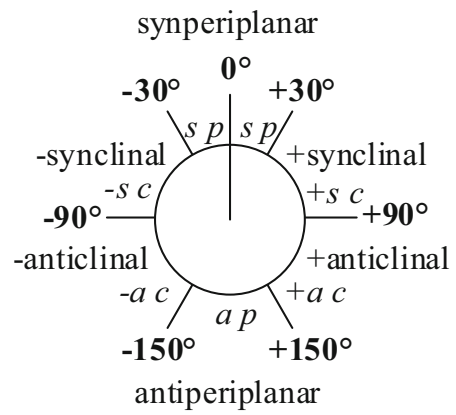

Fig. 10 IUPAC nomenclature of conformations (from Ref. [6]) 
Fig. 11 Conformations of butane

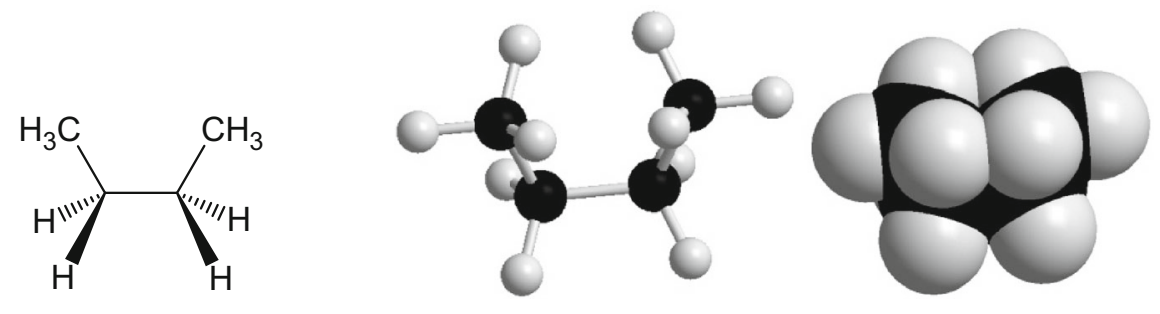

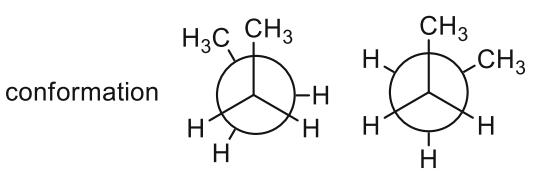

name

type of strain

$$
\text { syn }
$$

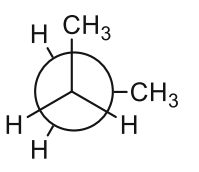

anticlinal

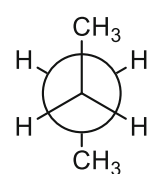

anti

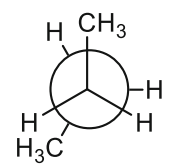

antinclinal

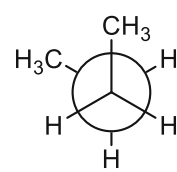

gauche

torsional and steric ("Me"-"H") steric

none torsional and

Fig. 12 Ball-and-stick and space-filling models of the eclipsing conformation of butane. Note that $\mathrm{C} 1$ and $\mathrm{C} 4$ hydrogen and carbon atoms "bump" into each other-there is not enough space for them. The result is steric strain
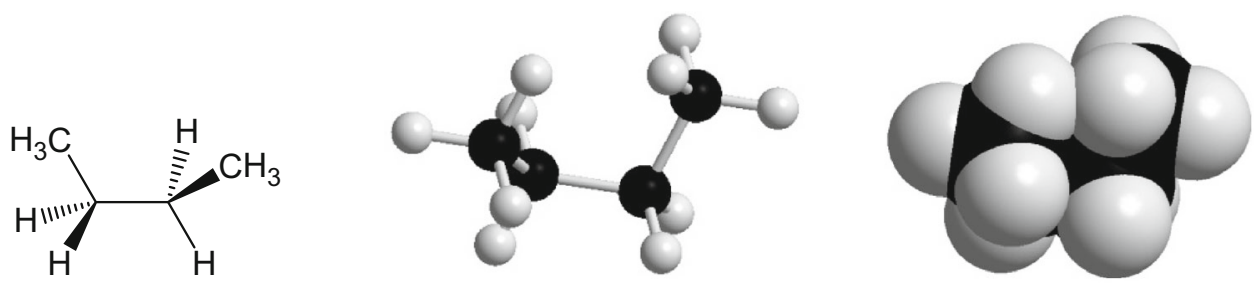

Fig. 13 Ball-and-stick and space-filling models of the gauche conformation of butane. Note that C1 and C4 hydrogen and carbons still "bump" into each other. Thus, like syn, this conformation exhibits steric hindrance

anticlinal, gauche conformations and finally, after completing full $360^{\circ}$ rotation, returning to the original syn conformation (Fig. 11).

A conformation where the methyl groups eclipse each other has a dihedral angle of $0^{\circ}$ (Fig. 12). This conformation is called syn, which is an abbreviation for synperiplanar. Such a conformation exhibits a high degree of strain. First, there is torsional strain, which is present in all the eclipsing conformations. Then, there is not enough room for both methyl groups, which are supposed to be in the same plane in this conformation. That strain is a result of van der Waals repulsion of groups that are close to each other and is called steric strain.

Rotation about the $\mathrm{C}^{2}-\mathrm{C}^{3}$ bond by $60^{\circ}$ results in a staggered conformation in which methyl groups are still relatively close to each other and there is considerable steric strain between them (Fig. 13). This conformation is called gauche conformation. Note that gauche conformation does not have torsional strain.

Rotation about the $\mathrm{C} 2-\mathrm{C} 3$ bond by another $60^{\circ}$ results in another eclipsing conformation (the dihedral angle is $120^{\circ}$ ). This conformation is called anticlinal. It has torsional strain and some steric strain that results from the eclipsing of methyl groups and hydrogen atoms. Since degree of steric strain is lower compared to eclipsing conformation with dihedral angle of $0^{\circ}$, the overall energy of this conformation is somewhat lower.

Rotation by further $60^{\circ}$, results in a staggered conformation where two methyl groups are $180^{\circ}$ apart (the dihedral angle is $180^{\circ}$ ). Therefore, the methyl groups are as far away from each other as possible. This conformation has no strain and is the conformation of the lowest energy. It is called the anti conformation (an abbreviation for antiperiplanar) of butane.

Continuing rotation by another $60^{\circ}$ results in a conformation with the dihedral angle of $240^{\circ}$. This conformation is a mirror image of the eclipsed anticlinal conformation with dihedral angle of $120^{\circ}$. Thus, the energies of the two conformations are the same. Rotation by another $60^{\circ}$ gives the other gauche conformation-the mirror image of the first one. Rotation by the final $60^{\circ}$ completes the full circle and results in the original eclipsing syn conformation.

The energy diagram below represents conformational analysis of butane (Fig. 14). Butane, as a more complex molecule than ethane, has an eclipsed conformation of the maximum energy and a staggered conformation of the minimum energy (anti conformation). There are also two eclipsed conformations (anticlinal), which represent local 


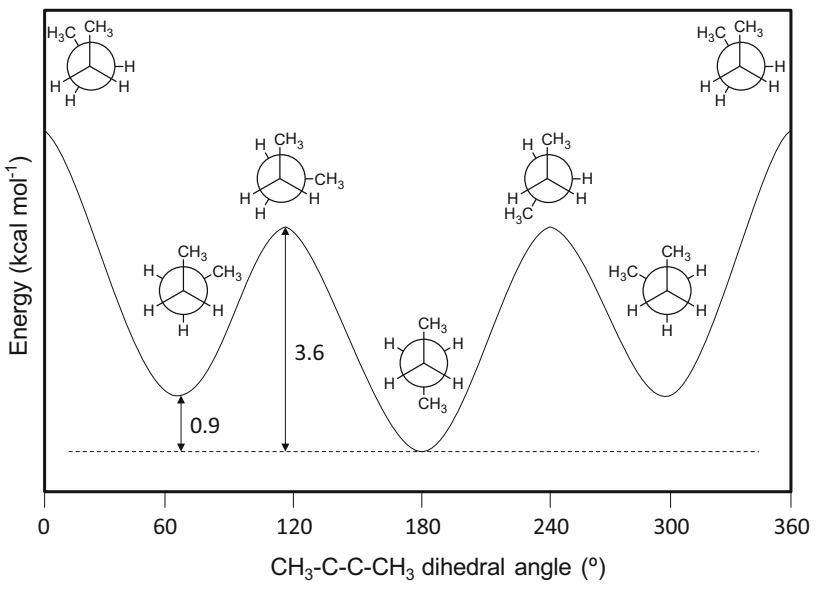

Fig. 14 Energy diagram for rotation about the carbon2-carbon3 bond in butane

energy maxima, and two staggered conformations (gauche), which represent local energy minima. Eclipsing conformations represent transition states between the staggered conformations. Note that gauche and anti conformations are special cases of staggered conformations. In conclusion, butane, in addition to the torsional strain, may exhibit steric strain.

Even though anti conformation is the lowest energy conformation of butane, at room temperature butane does not exist exclusively in the anti conformation. There are two gauche conformations and one anti. Thus, probability factors favor a gauche conformation. Furthermore, a mixture of conformations is favored since it has greater entropy compared to a single conformation. Preference for a mixture and the increased proportion of gauche conformation increases with temperature.

\section{Types of strain}

Conformational analysis of simple alkanes revealed two types of strain-torsional and steric. According to Hendrickson [7] and separately Allinger [8] the total strain of a conformation is the sum of:

1. Bond strain-stretching or compression of chemical bonds. This type of strain is rather severe and is not encountered very often in organic compounds. To minimize bond strain, a molecule adopts conformations that have other, less energy-demanding, types of strain.

2. Torsional strain (eclipsing strain, Pfitzer strain) is caused by eclipsing interactions. Torsional strain is considerably higher compared to steric or angle strain, which are explained next.

3. Steric strain (Van der Waals strain, Prelog strain) is caused by atoms forced too close to each other.
Transannular strain (Prelog strain) is a form of steric strain characteristic of medium rings.

4. Angle strain (Bayer strain, classical strain) is a result of deviation from the ideal bond angle. Compared to other types of strain, increase in energy of a conformation caused by angle strain is relatively low. As a result, a molecule can accommodate relatively large deviation from an ideal bond angle and still be stable.

Any cyclic molecule tends to assume such a conformation in which the sum of the four types of strain is minimal. Since bond strain is considerably higher than the rest, it is relatively rare in cyclic compounds. If possible, a molecule will assume a conformation that exhibits one or more of the other three types of strain to avoid bond strain. Therefore, in most cases the overall strain is the interplay of the remaining three types of strain that are responsible for the energy of a particular conformation.

\section{Classification of cyclic compounds}

Cyclic compounds are classified, according to the number of atoms in the ring and properties, as small rings with 3 or 4 atoms, common rings with $5-7$, medium rings $8-11$, and large rings with 12 or more. Rings of similar size exhibit similar properties.

Small rings are rigid and highly strained. They are characterized both by a large deviation from the ideal tetrahedral valency angle- $\mathrm{a}$ high angle strain, and eclipsing interactions-a high torsional strain. Common rings are characterized by the tetrahedral valency angles and having substituents pointing only "out" of the ring. Medium rings are characterized by a specific type of steric strain called transannular interactions. It is a strain caused by substituents pointing "into" the ring. Large rings usually exhibit very little strain and resemble the corresponding open-chain compounds.

Sometimes small and common rings are grouped together into classical rings while medium and large rings are grouped into many-membered rings. Classical rings are relatively easy to form. Even though some may be rather strained, entropy of formation is favorable as the two ends of the chain are close together. Many-membered rings are considerably more difficult to prepare due to unfavorable entropy of activation.

\section{Cyclohexane}

\section{Conformations of cyclohexane}

It may be convenient to start analysis of cycloalkanes with cyclohexane rather than a chronological examination of the 
Fig. 15 a Cyclohexane as a planar molecule. b Chair conformation of cyclohexane

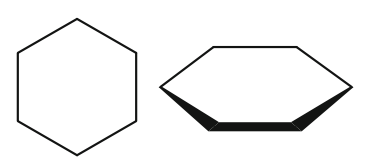

a<smiles>C1C2CC3CC1C3C2</smiles>

b

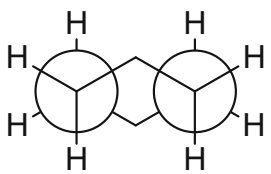

b rings from the smallest to the largest. Cyclohexane is the smallest ring that exhibits no strain and types of bonds and conformations of cyclohexane form the basis of conformational analysis of other rings.

In nineteenth century, von Baeyer represented cyclohexane as a planar molecule (Fig. 15a). Baeyer's description of cyclohexane was apparently a result of the consideration of an inadequate type of molecular models (Kekulé-von Baeyer models) [9]. Those models had very long carbon-carbon bonds and with them one was able to make large planar rings. In the von Baeyer model of cyclohexane, there is some angle strain and a rather high degree of torsional strain.

In a hypothetical "planar cyclohexane" $\mathrm{C}-\mathrm{C}-\mathrm{C}$ bond angles would be larger $\left(120^{\circ}\right)$ than tetrahedral. Furthermore, such conformation would exhibit 12 pairs of eclipsing $\mathrm{C}-\mathrm{H}$ interactions. Instead, cyclohexane ring puckers to relieve both the torsional and the angle strain. In fact, one of the resulting puckered cyclohexane conformations has no strain at all. The two extreme conformations of cyclohexane are chair and boat. Chair conformation is the one without strain (Fig. 15b). In a chair conformation all of the bond angles are tetrahedral angles and all of the $\mathrm{C}-\mathrm{H}$ bonds are in staggered conformations.

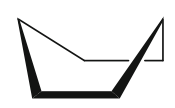

a

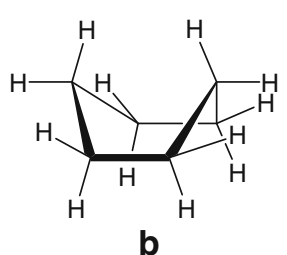

b

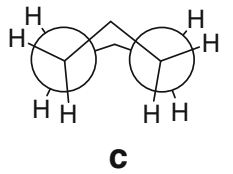

C
Fig. 16 Boat conformation of cyclohexane a side-on perspective view, b with hydrogens represented, c Newman projection
In a boat conformation there are a total of four pairs of eclipsing $\mathrm{C}-\mathrm{H}$ interactions (Fig. 16). Thus, boat conformation of cyclohexane has a high torsional strain. The best way to see that is by viewing the cyclohexane molecule in a Newman projection (Fig. 16c). Additional strain is caused by the two "flagpole" hydrogens, which project to the inside of the ring. They are so-called "intraannular" substituents and they cause a form of steric strain called transannular strain. However, in cyclohexane that strain is negligible. It is the high torsional strain in a boat conformation that makes it so unstable that it is considered to be a transition state rather than an actual conformation.

A more stable conformation is a twist-boat (Fig. 17). Thus, the boat "twists" to reduce the transannular and, considerably more serious, torsional strain. However, twisting of the ring only places adjacent $\mathrm{C}-\mathrm{H}$ bonds in a skew conformation and only reduces but does not entirely eliminate the torsional strain.

\section{Axial and equatorial bonds of cyclohexane}

In a chair conformation of cyclohexane one can identify two types of carbon-hydrogen bonds: axial and equatorial. Axial bonds are parallel to the threefold axis of symmetry of the ring, while equatorial are distributed around the ring's “equator” (Fig. 18).

\section{Bond angles of cyclohexane}

The ideal tetrahedral bond angle is $109.5^{\circ}$ only if the carbon atom is bonded to four identical substituents (for example: $\mathrm{CH}_{4}$ or $\mathrm{CCl}_{4}$ ). If the substituents are different,

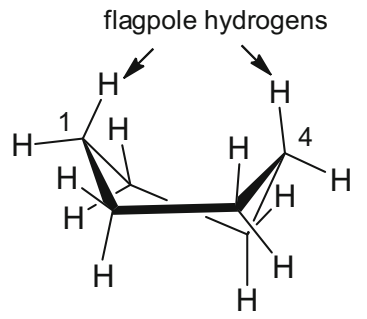

Perspective formula

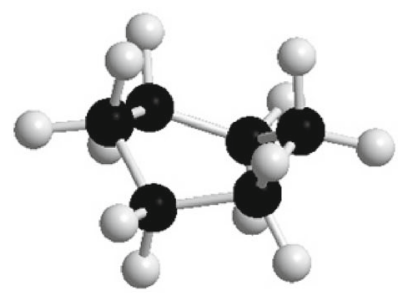

Ball and stick model

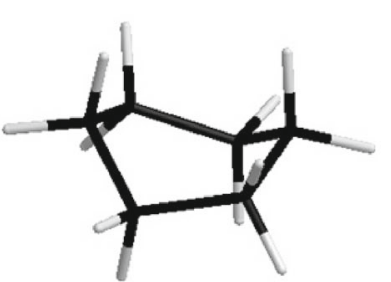

Wire frame model

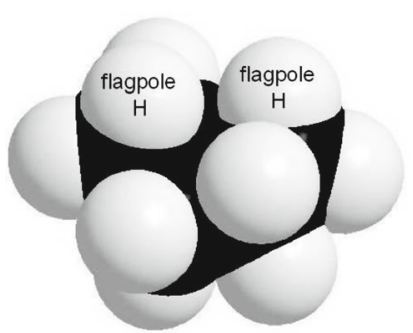

Space filling model

Fig. 17 Twist-boat conformation of cyclohexane 
Fig. 18 Axial and equatorial bonds of cyclohexane

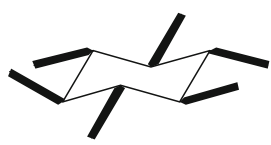

equatorial bonds
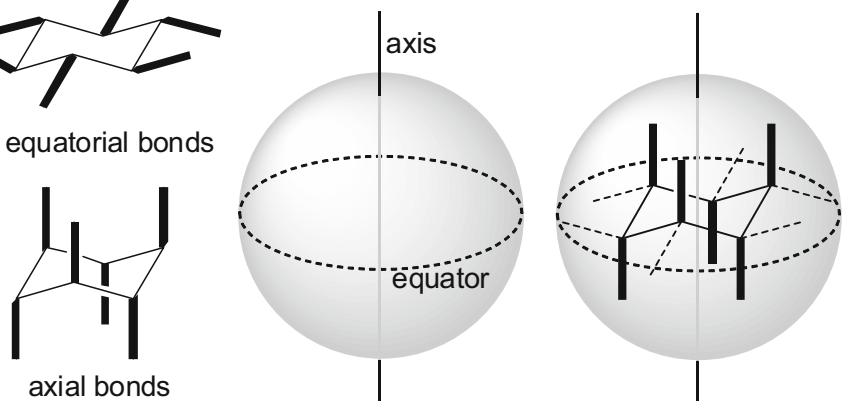

Axial bonds (bold) are parallel to the ring axis.

Equatorial bonds (dashed) are distributed around the equator of the ring.
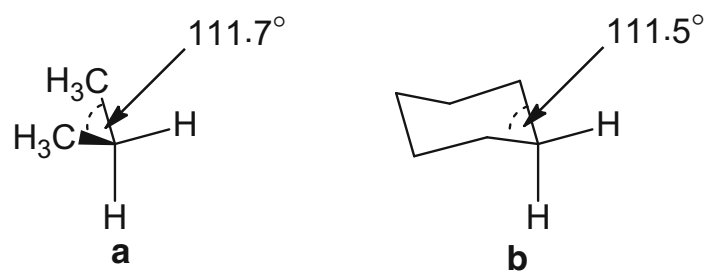

Fig. 19 Bond angles in cyclohexane

the bond angle deviates to accommodate different steric requirements of different substituents. Thus, in propane the $\mathrm{C}-\mathrm{C}-\mathrm{C}$ bond angle is $111.7^{\circ}$. Comparison of cyclohexane to propane is appropriate as in each molecule carbon atoms have identical sets of four substituents- two carbon and two hydrogen atoms (Fig. 19). In a chair conformation of cyclohexane twisting of the ring results in a bond angle of $111.5^{\circ}$, which is very close to that of propane and, therefore, cyclohexane does not exhibit an angle strain.

\section{Drawing of the chair conformations of cyclohexane}

It is important to draw cyclohexane conformations correctly - with correct bond angles and correct placement of the axial and equatorial bonds. One way to draw the cyclohexane ring and its equatorial and axial bonds is shown below (Fig. 20) [10].

One should start by drawing a five-atom chain in a zigzag conformation a. The conformation resembles letter M. One should keep in mind that the drawing should represent a side-on perspective view of the ring. Thus, the angles in the chain should actually be $135^{\circ}-150^{\circ}$. Next, one draws another five-atom chain parallel to the first one in a form of a letter $\mathrm{W}$ as in $\mathrm{b}$. The starting point is approximately one half bond length below the midpoint of the first bond in $\mathrm{M}$. The first bond in $\mathrm{W}$ should be parallel to the second bond in M. Then, the second atom of the first chain should be connected to the second atom of the second chain and the second last atom of the first chain should be
Fig. 20 Drawing of chair conformations of cyclohexane

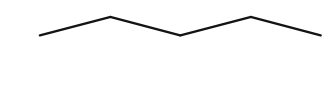

a

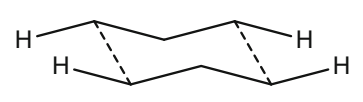

d
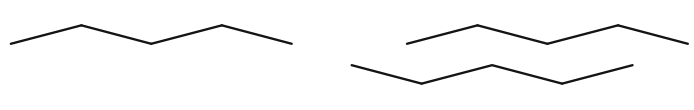

h

g

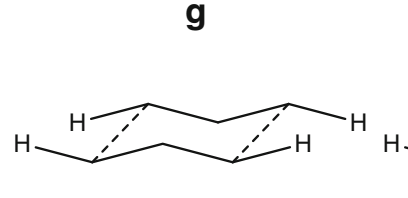

j

b

e

k
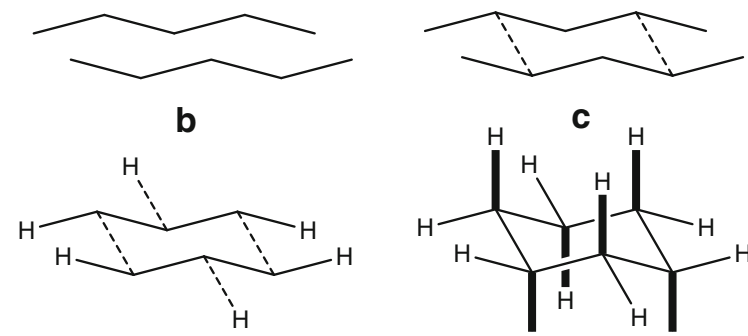

C

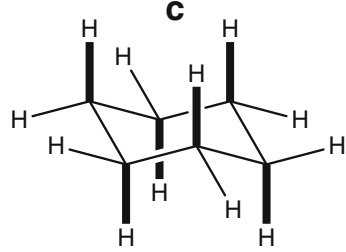

f

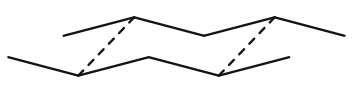

i

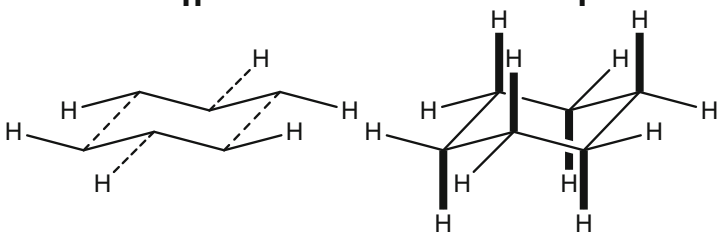


Fig. 21 Inversion of cyclohexane chair conformation

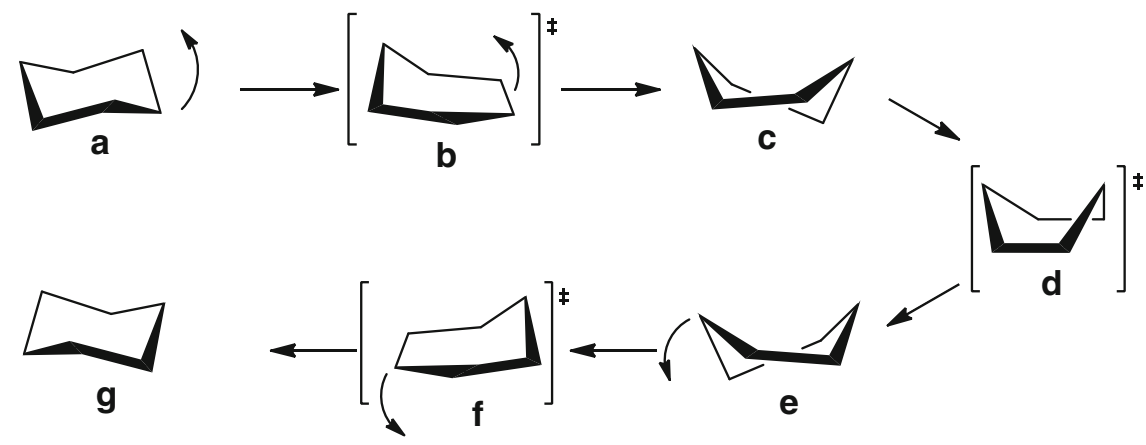

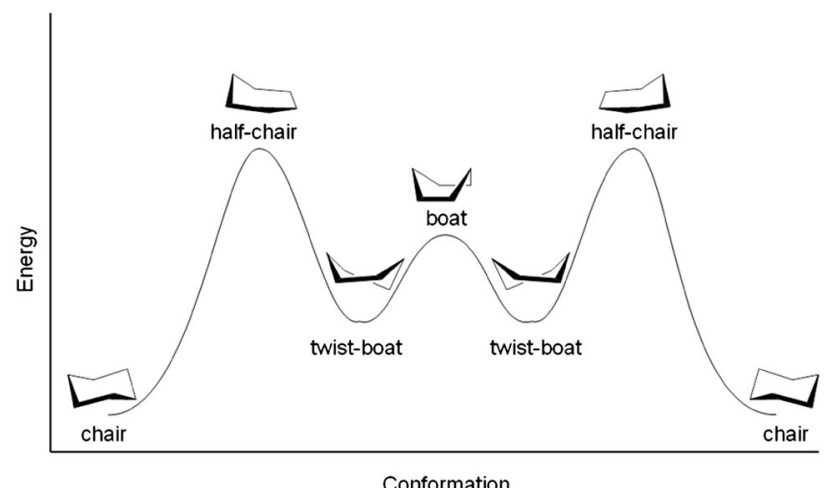

Fig. 22 Energy diagram for conformations of cyclohexane

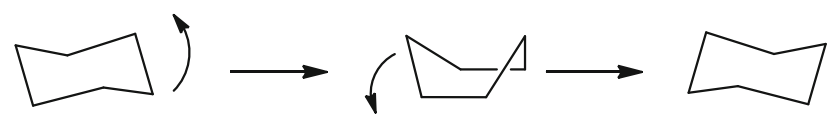

Fig. 23 Chair flip using molecular models

connected to the second last atom of the second chain c. As an aid in determining which atoms to connect, one can place hydrogen atoms at the ends of each chain and then connect the terminal carbon atoms $\mathrm{d}$. The resulting drawing depicts all carbon-carbon bonds and four of the six equatorial carbon-hydrogen bonds. The remaining two equatorial bonds are parallel to the two $\mathrm{C}-\mathrm{C}$ bonds just drawn e. The axial bonds are parallel to the edge of the paper as in $\mathrm{f}$. Sequence $\mathrm{g}-1$ shows the drawing of the alternate chair conformation. One should start by drawing a five-atom chain in a zigzag conformation $g$ (identical to a). Next, one draws another five-atom chain parallel to the first one in a form of a letter $\mathrm{W}$ with the starting point now approximately one half bond length below the midpoint of the last bond in $M(h)$. The rest of the drawing follows the same process as for the first chair. The second atom of the first chain should be connected to the second atom of the second chain and the second last atom of the first chain should be connected to the second last atom of the second chain (i), terminal bonds are labeled as the equatorial hydrogens (j) and the remaining two equatorial bonds are drawn parallel to the two $\mathrm{C}-\mathrm{C}$ bonds just drawn (k). The axial bonds are parallel to the edge of the paper as in 1 .

\section{Interconversions of cyclohexane conformations}

There are two chair conformations of cyclohexane. The two conformations are mirror images of each other and, therefore, have the same energy. At room temperature cyclohexane molecules undergo an interconversion (a "flip") from one chair conformation to another. In cyclohexane interconversion of configuration proceeds through a mechanism described as an inversion.

An inversion of a cyclohexane chair involves all of the conformations of cyclohexane (Fig. 21). Starting with a chair conformation a, cyclohexane first passes through a "half-chair" conformation b. The half-chair, as expected, has a very high energy and is only a transition state, which gives rise to the actual conformation-twist-boat c. Next, the twist-boat conformation flips into the alternative twistboat conformation e. The transition state for this flip is the boat conformation $\mathrm{d}$. Then, the cyclohexane molecule passes through another half-chair transition state $\mathrm{f}$ (mirror image of the first one) and finally arrives at the alternative chair conformation $\mathrm{g}$.

The relative energies of cyclohexane conformations are shown in Fig. 22. The two chairs (a and g) and the two twist-boats (c and e) are the actual conformations. Halfchairs (b and f) and boat (d) are only transition states.

With a molecular model of cyclohexane, one performs an abbreviated version of the chair flip (Fig. 23). When using molecular models, the twist-boat conformation is not necessary for a chair flip and it is not obvious why it should be a part of the process. A problem with molecular models is that they do not distinguish between a conformation and a transition state and with them one can go directly from one conformation to another without having to pass through the transition states and the intermediates. Students should understand that molecular models are just thatmodels. They are not actual molecules.

Calculations show that twist-boat conformation is necessary for a chair flip (Fig. 24) [11]. However, for the chair 


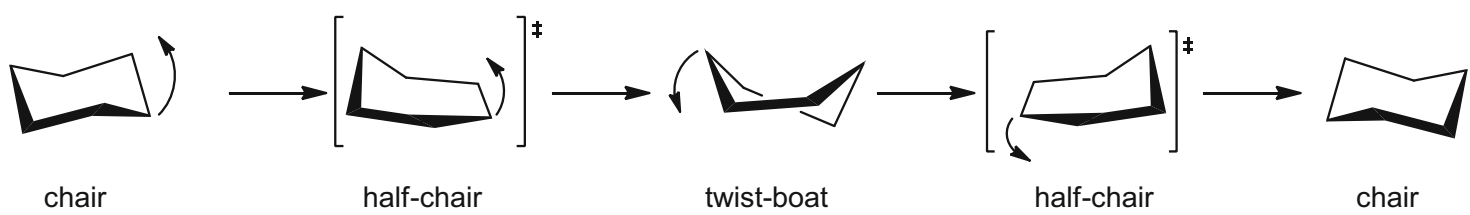

Fig. 24 Chair flip of cyclohexane (from Ref. [11])

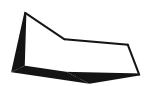

a

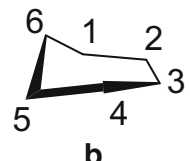

b

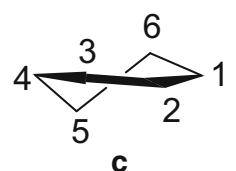

Fig. 25 a Stylized representation of half-chair. b Its actual shape. c View from a different angle

flip to occur, cyclohexane does not have to pass through a boat conformation.

A half-chair conformation is illustrated above as having five carbon atoms in a plane for clarity (Fig. 25a). Actual half-chair conformation has only four carbon atoms in the same plane (Fig. 25b) [12]. This is better observed from a different angle (Fig. 25c). Conformation represented by Fig. 25a is sometimes referred to as an envelope configuration to distinguish it from a half-chair.

\section{Cyclohexane is not strain free}

Cyclohexane is commonly referred to as being a strain-free ring in its chair conformation. However, strictly speaking that is not the case. Cyclohexane exhibits some steric strain. Obviously four carbon sequences in a cyclohexane chair are forced into gauche conformations. Therefore, the same type of strain present in a gauche conformation of butane is present in cyclohexane. Even though we understand that the chair conformation of cyclohexane is not entirely strain free, it is a commonly used "strain-free" standard to which stabilities of other cycloalkanes are compared.

A definition of what is strain free and what represents an appropriate comparison is not always clear. For example, in hexane gauche conformation is predominant as there are many gauche conformations and only one anti. Thus, when comparing cyclohexane with a hexane, one can say that it has about the same energy and is strain free. However, cyclohexane has a considerably higher energy compared to an all-anti conformation of hexane.

All rings, except for cyclopropane, suffer from 1,3-interactions. It is the same interaction that raises the energy of a staggered conformation of propane. In cyclohexane it is called 1,3-diaxial strain. To minimize it, the molecule flattens a bit (valence angle of ca. $112^{\circ}$ ) and the axial bonds move a bit from vertical orientation and slightly apart from each other. Thus, the actual conformation of the chair of

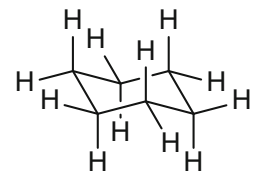

a

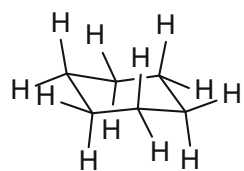

b
Fig. 26 a Idealized representation of cyclohexane chair with valence angle of $109^{\circ} 28^{\prime}$ and vertical axial bonds. b A more realistic representation

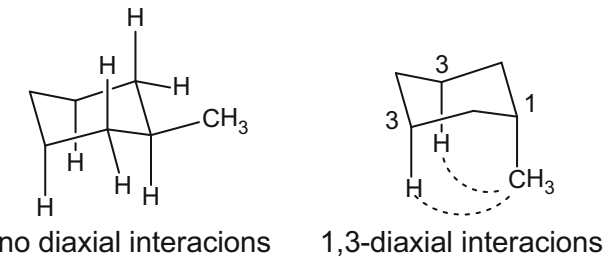

Fig. 27 a 1,3-Diaxial interactions are present only in conformer that has axial substituent. b A conformation in which the substituent is equatorial is more stable

cyclohexane is a bit different from the common, idealized, representation (Fig. 26). With hydrogens as substituents, magnitude of 1,3-interaction is rather small and the calculation of such strain is included in the valence angle distortion energy. With larger substituents its magnitude increases and it is the principal factor responsible for steric strain in substituted cyclohexanes.

\section{Monosubstituted cyclohexanes}

Two alternate chair conformations of cyclohexane have the same energy. However, that may not be the case if cyclohexane is substituted. When one chair conformation of a substituted cyclohexane flips into the alternative chair conformation, an axial substituent becomes equatorial and vice versa (Fig. 27). A chair conformation with an axial substituent is less stable compared to a chair conformation with an equatorial substituent. The reason for lower stability is in steric interactions between the axial substituent and the axial hydrogens on the same side of the ring (Fig. 27a). If the carbon atom with the axial substituent is numbered as carbon 1, then each carbon that has axial hydrogen on the same side of the ring can be numbered as carbon 3 (in one case the numbering is clockwise and in the 
other counterclockwise). Steric interactions between the axial substituent and the axial hydrogens are called 1,3diaxial interactions. Therefore, as a general rule, a conformation in which the substituent is equatorial is more stable (Fig. 27b).

1,3-Diaxial interaction in cyclohexane is a special case of a gauche interaction. In this case the molecule is "frozen" in a gauche conformation as the rotation about the $\mathrm{C}-$ $\mathrm{C}$ bond is not possible. Methyl substituent and carbons 1-3 make up the "butane" part of the cyclohexane responsible for the gauche interaction (Fig. 28).

\section{Conformations of disubstituted cyclohexanes: cis-trans isomerism}

Disubstituted cyclic compounds exhibit cis-trans isomerism. In a cis isomer, both substituents are on the same side of the ring, while in a trans isomer the two substituents are on the opposite sides (Fig. 29). Cis-trans isomerism is a consequence of restricted rotation about carbon-carbon bonds in cyclic compounds. Note that in a cis isomer both substituents point "up" (or "down") and that in a trans

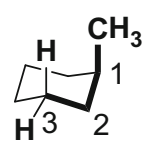

1,3-diaxial interacion

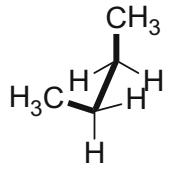

gauche conformation of butane
Fig. 28 1,3-Diaxial interaction in cyclohexane is a special case of a gauche interaction isomer one substituent points "up" and the other one "down". The chair flip does not change the orientation of substituents. One that was pointing "up" still points "up" and the one that was pointing "down" still points "down". However, chair flip changes conformation of a substituent from equatorial into axial and vice versa.

In a disubstituted cyclohexane, the most stable conformation is the one in which the largest number of substituents are in equatorial positions. If the number of equatorial and axial substituents of the same size in two conformations is the same, then the two conformations are equally stable. Examples shown below illustrate this concept. The arrows indicate the position of the equilibrium with the longer arrow pointing to the more stable isomer while the arrows of the same length indicate isomers of equal stability (Fig. 30). cis-1,3-Dimethylcyclohexane has a low-energy diequatorial and a high-energy diaxial conformations. trans-1,3-Dimethylcyclohexane has two equivalent conformations in each of which one substituent is equatorial and the other one axial. cis-1,4-Dimethylcyclohexane has two equivalent conformations in each of which one substituent is equatorial and the other one axial. trans-1,3-Dimethylcyclohexane has a low-energy diequatorial and a high-energy diaxial conformations.

Diequatorial conformation of trans-1,2-dimethylcyclohexane is a special case as in this molecule there is steric strain. It is another gauche interaction (Fig. 31). In diaxial conformation four 1,3-diaxial interactions are present. Thus, despite some steric strain, diequatorial conformation is still more stable compared to a diaxial. The diequatorial trans isomer is also lower in energy compared to the cis
Fig. 29 Cis and trans isomers of dimethylcyclohexanes

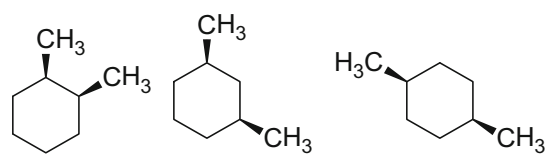

cis isomers

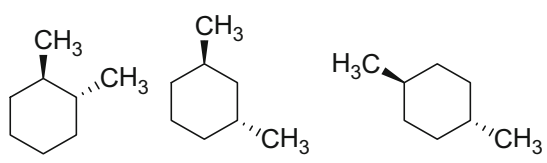

trans isomers
Fig. 30 Conformations of 1,3and 1,4-dimethylcyclohexanes

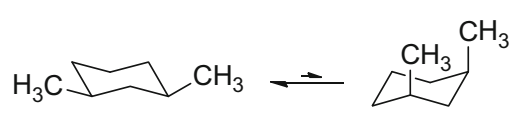

cis-1,3-dimethylcyclohexane

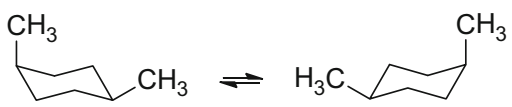

cis-1,4-dimethylcyclohexane

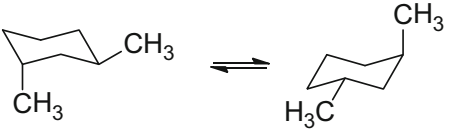

trans-1,3-dimethylcyclohexane<smiles>CC#CC1(C)CCC(C)CC1</smiles>

trans-1,4-dimethylcyclohexane 
Fig. 31 Conformations of 1,2dimethylcyclohexane

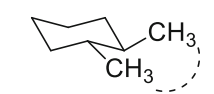

Gauche interaction between two methyl substituents

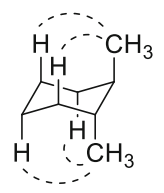

Four 1,3-diaxial interactions

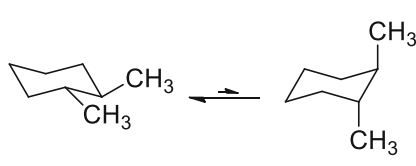

trans-1,2-dimethylcyclohexane

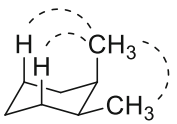

Three gauche interactions in the cis isomer



cis-1,2-dimethylcyclohexane

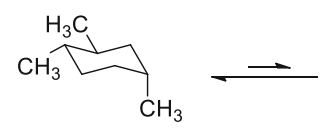

more stable - two equatorial substituents

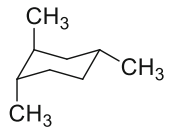

less stable - two axial substituents

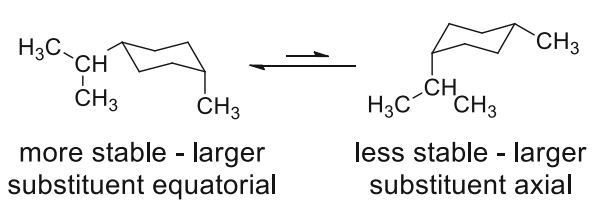

b

Fig. 32 a In the most stable conformation the largest number of substituents are in equatorial positions. b When substituents are of different sizes the larger substituent occupies an equatorial position

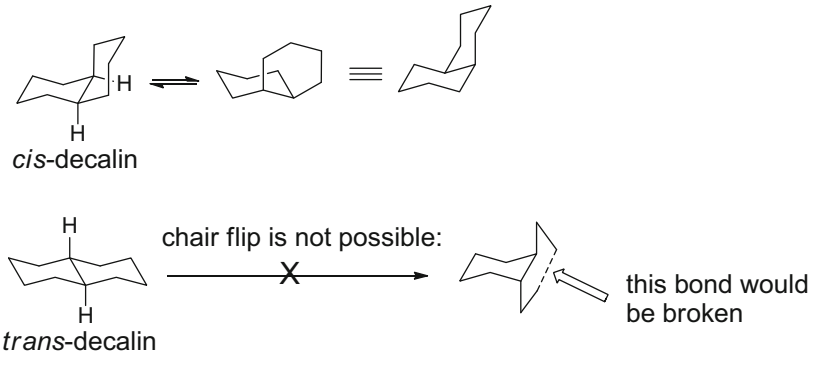

Fig. 33 Conformations of two isomeric decalins

isomer as only one gauche interaction is present compared to three (one gauche and two 1,3-diaxial) in the cis-1,2dimethylcyclohexane. As diequatorial conformations of cis-1,3-dimethylcyclohexane and trans-1,4-dimethylcyclohexane exhibit no steric hindrance, they are lower in energy compared to the diequatorial conformation of trans1,2-dimethylcyclohexane.

In polysubstituted cyclohexanes, the most stable conformation is the one in which the largest number of substituents are in equatorial positions (Fig. 32a). If the two substituents are of different sizes, then, in a more stable conformation, the larger substituent occupies the equatorial position (Fig. 32b).

\section{Fused six-membered rings: decalin}

Two fused six-membered carbon rings form decalin (bicyclo[4.4.0]decane). Decalin exhibits a cis-trans isomerism (Fig. 33). cis-Decalin has two hydrogens at angular positions (those where the two rings meet) on the same side, while trans-decalin has the two angular hydrogens on the opposite sides of the ring. Both cis-decalin and trans-decalin have two fused cyclohexane rings in chair conformations. Note that only cis-decalin is capable of a chair flip. In trans-decalin, a chair flip would result in two axial carbon-carbon bonds with the remaining bonds too far apart to close a cyclohexane ring.

\section{Small rings}

\section{Cyclopropane}

The simplest cycloalkane is cyclopropane. A molecule of cyclopropane is planar and has the shape of an equilateral triangle (Fig. 34a). That is obvious since three points in space define a plane (unless all three points are co-linear). Thus, cyclopropane has only one conformation and hence there is no conformational analysis. Since the cyclopropane molecule is planar, all carbon-hydrogen bonds are eclipsing (Fig. 34b). Note that there is no possibility for bond rotation in cyclic compounds. Thus, this molecule also has a high degree of torsional strain. In cyclopropane, bond angles are $60^{\circ}$. Therefore, there is a large deviation from the tetrahedral bond angle and, as a result, there is a high degree of angle strain. If bonding electron pairs in cyclopropane were along the internuclear axes (shown as a dashed bond in Fig. 34d), the angle strain would be too 


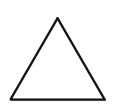

a

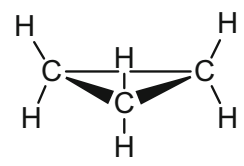

b

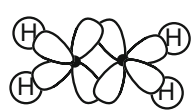

C

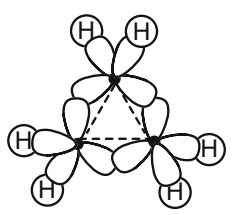

d



e

Fig. 34 a Line formula of cyclopropane. b Perspective formula of cyclopropane. c Pauling model of ethene. d Bent bonds of cyclopropane. e Walsh bonding model of cyclopropane with hybrid $s p^{2}$ orbitals shown in grey and unhybridized $p$ in white

Fig. 35 Conformation and bonds of cyclobutane
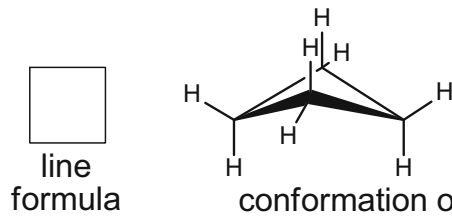

conformation of cyclobutane
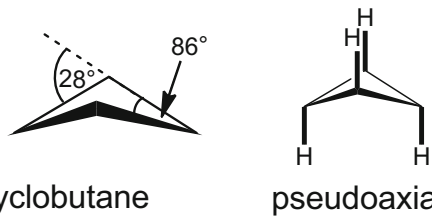

pseudoaxial bonds

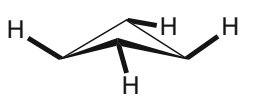

pseudoequatorial bonds large. In an energetically more favorable arrangement, the electron pairs form "bent" or "banana" bonds. Bent bonds were introduced by Linus Pauling in 1931 to describe bonding in alkenes (Fig. 34c) [13]. "Banana" bonds are generally considered to result from an overlap of carbon $s p^{3}$-hybridized orbitals (Fig. 34d) [14]. However, model proposed by Walsh involves $s p^{2}$-hybridized carbon atoms and carbon-carbon bonds are formed by the overlap of unhybridized $p$ orbitals (Fig. 34e) [15]. From X-ray diffraction studies, the carbon-carbon bond length was found to be $1.51 \AA$ instead of $1.54 \AA$. That is viewed as a support for banana bonds model. However, a shorter bond is also consistent with Walsh model (shorter bonds due to $s p^{2}$ hybridization of carbon atom).

A bent bond results in a reduced angle strain, but, since the orbital overlap is not as good as in an ordinary $\sigma$ bond, the bond is considerably weaker. While strength of an ordinary carbon-carbon bond (for example carbon-carbon bond in ethane) is ca. $83-85 \mathrm{kcal} \mathrm{mol}^{-1}$ (345-355 $\mathrm{kJ} \mathrm{mol}^{-1}$ ), the strength of carbon-carbon bond in cyclopropane is only ca. $65 \mathrm{kcal} \mathrm{mol}^{-1}\left(272 \mathrm{~kJ} \mathrm{~mol}^{-1}\right)$. Energy of a carbon-carbon $\pi$ orbital is ca. $60-65 \mathrm{kcal} \mathrm{mol}^{-1}$ $\left(251-272 \mathrm{~kJ} \mathrm{~mol}^{-1}\right)$. This is an important consideration since sometimes the reactivity of cyclopropane resembles that of an alkene. In fact, there is a view that a double bond can be considered to be a two-carbon ring. Unlike other cycloalkanes, and alkanes in general, cyclopropane is very reactive since a reaction that results in a ring opening relieves both the angle and the torsional strain.

While cyclopropane exhibits considerable strain, there is one type of strain that is absent. Since all $\mathrm{C}-\mathrm{H}$ bonds on different carbons are 1,2 relative to each other, in cyclopropane 1,3-repulsive interactions are not possible. All other cycloalkanes exhibit 1,3-repulsive interactions.

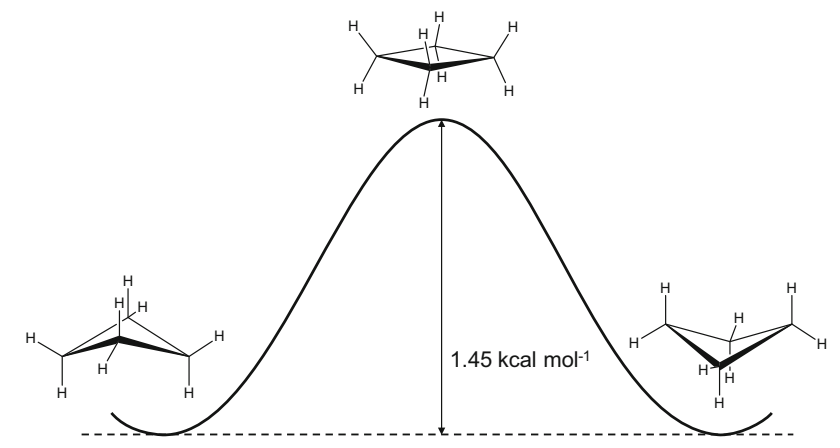

Fig. 36 Inversion of cyclobutane

\section{Cyclobutane}

A molecule of cyclobutane adopts a puckered conformation to minimize torsional strain. If it were planar, there would be eight pairs of $\mathrm{C}-\mathrm{H}$ eclipsing interactions and the torsional strain would have been rather high. In a puckered conformation, $\mathrm{C}-\mathrm{H}$ bonds are in a skew conformation and, therefore, some torsional strain is still present. On the other hand, cyclobutane still has a relatively high angle strain. Bending of the ring increases its angle strain. The dihedral angles are ca. $28^{\circ}$ and the valency angles are $86^{\circ}$ (instead of $90^{\circ}$ in a planar square) (Fig. 35). However, the angle strain is not as high as in the case of cyclopropane. As one can see from the example of cyclobutane, in a cyclic molecule a high angle strain is less destabilizing compared to a high torsional strain.

One can identify two types of $\mathrm{C}-\mathrm{H}$ bonds in cyclobutane: pseudoaxial and pseudoequatorial (Fig. 35). Pseudoaxial are parallel to the imaginary axis of the ring, while pseudoequatorial are distributed around the rings "equator". Pseudoaxial and pseudoequatorial bonds of cyclobutane resemble to axial and equatorial bonds of 
Fig. 37 Conformations of cyclopentane

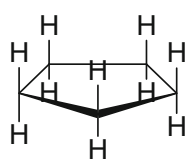

Haworth formula
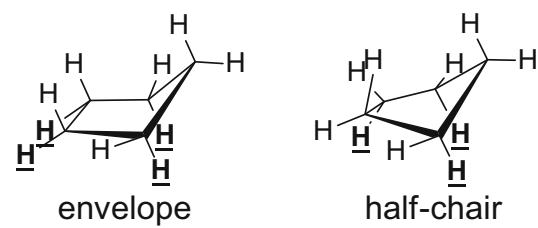

Fig. 38 a Conversion of an envelope conformation into a half-chair. b Conversion of a half-chair conformation into an envelope
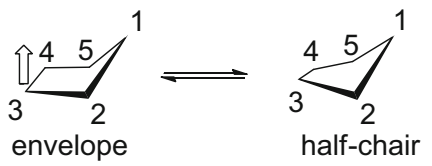

a

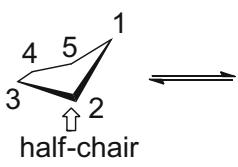

half-chair

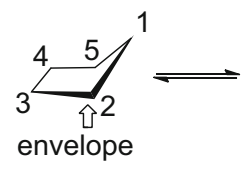

b cyclohexane. As in cyclohexane, conformation with substituents in pseudoequatorial positions is more stable except that the energy differences are smaller.

Cyclobutane is a ring that undergoes a true inversion of conformation. It means that one puckered conformation is interconverted into another by passing through a transition state in which the ring is planar (Fig. 36). Inversion processes in larger rings are usually composed of a sequence of unsymmetric step processes and do not involve a planar transition state. The barrier to inversion in cyclobutane is $1.45 \mathrm{kcal} \mathrm{mol}^{-1}\left(5.5 \mathrm{~kJ} \mathrm{~mol}^{-1}\right)$.

\section{Common rings}

\section{Cyclopentane}

Angles in a regular pentagon are $108^{\circ}$. That is very close to the ideal tetrahedral angle of $109.5^{\circ}$ and, therefore, if cyclopentane were planar it would not exhibit any significant angle strain. However, in a planar cyclopentane there would be a high degree of torsional strain. All ten of the carbon-hydrogen bonds would be in eclipsing conformations. This is obvious from the Haworth formula (Fig. 37). A Haworth formula represents the ring as being planar and all the bonds to the substituents are represented as being vertical.

As in the case of cyclobutane, a molecule of cyclopentane is not planar. The overall strain is reduced by twisting of the molecule out of the plane, but the angle strain is increased since valency angles become considerably smaller than $108^{\circ}$. Thus, in cyclobutane and cyclopentane puckering of the molecule results in an increased angle strain. The $\mathrm{C}-\mathrm{C}-\mathrm{C}$ bond angle is already smaller than the ideal tetrahedral angle and puckering further reduces it. As the resulting structure is no longer symmetrical, bond angles vary from $102^{\circ}$ to $106^{\circ}$ [16]. Preferred conformations of cyclopentane are the envelope and the half-chair (also called the "twist" form). In an envelope conformation four carbon atoms are in the same plane and one is either above or below it. In a half-chair conformation, three carbon atoms are in a single plain, while two non-adjacent carbon atoms are either above or below it. Thus, when one places a model of an envelope conformation on the table, four hydrogen atoms (shown in bold and underlined) will rest on the table top. When one places a model of a halfchair conformation on the table, three hydrogen atoms will rest on the table top. Note that either of the two conformations still has some torsional strain as in cyclopentane some eclipsing interactions cannot be avoided. In conclusion, cyclopentane has torsional strain and a very small angle strain.

Unlike cyclohexane and cyclobutane where interconversion of conformations proceeds through inversion, in cyclopentane conversion of an envelope into a half-chair proceeds through a mechanism described as pseudorotation (Fig. 38). A conversion of an envelope into a half-chair and vice versa involves only a small movement of a single carbon atom. It is considered that there is no energy barrier for this process. Starting with an envelope conformation, any one of carbon atoms except for the "flap" carbon moves up or down. If the "flap" atom is labeled as 1, then movement of either atom 3 or 4 in the direction of the flap converts an envelope into a half-chair. Of course, movement of one carbon atom also causes slight movement of the remaining carbons. Starting with a half-chair, a half way up or down movement gives the corresponding envelope. Continuation of that movement in the same direction gives the alternate, mirror image, half-chair.

There are a total of 10 envelope and 10 half-chair conformations. Cyclopentane undergoes conformational change from an envelope as shown in Fig. 39. Small arrows indicate the carbon that moves and the direction of the move. It appears as if the sequence of movements of each atom is rolling like a wave around the ring and hence the name pseudorotation. Pseudorotation, unlike inversion, proceeds without passing through a significant energy barrier. 


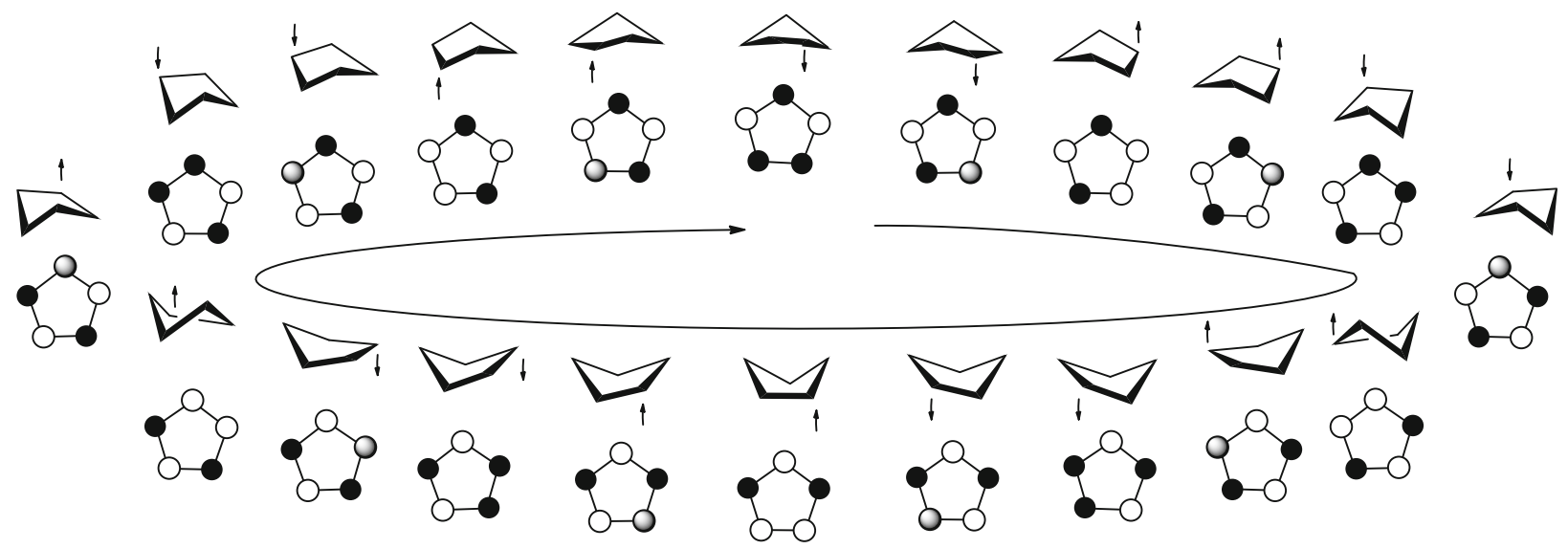

Fig. 39 Pseudorotation of cyclopentane. The top structures are side view perspective drawings with small arrows indicating the carbon atom that moves and the direction of the move. The bottom structures

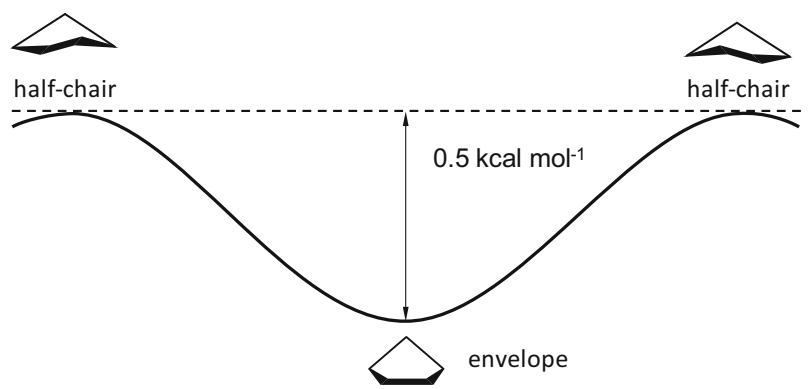

Fig. 40 Interconversions of conformations of cyclopentane. Envelope is only a slightly more stable compared to a half-chair and there is virtually no barrier to intercoversion

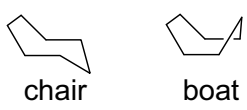

a

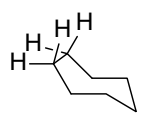

b
Fig. 41 a Chair and boat conformations of cycloheptane. b Torsional strain in each conformation. Flagpole hydrogen, responsible for steric strain, is shown in red

The difference in energy between the two conformations of cyclopentane is slight, the envelope form being the more stable by about $0.5 \mathrm{kcal} \mathrm{mol}^{-1}$ [17] (Fig. 40). Planar conformation is about $5 \mathrm{kcal} \mathrm{mol}^{-1}$ higher in energy [18].

\section{Cycloheptane}

Cycloheptane is the largest common ring and it exhibits some features associated with medium rings such as a more difficult preparation and steric strain in some conformations. As an odd-membered ring, it cannot have a strainfree conformation. There are two families of conformations of cycloheptane-chair and boat (Fig. 41a). Unlike cyclohexane, the chair conformation is a part of a family of represent view from the top. Black circles represent atoms above the molecular plane, white below and grey in the plane

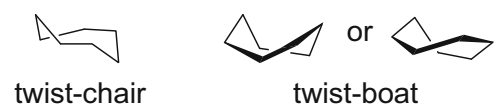

Fig. 42 Low-energy conformations of cycloheptane

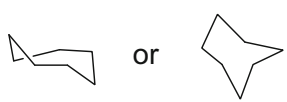

twist-chair<smiles>CC1(C)CCCCC1</smiles>

1,1-dimethylcycloheptane
Fig. 43 In the lowest energy conformation a geminally disubstituted cycloheptane has both substituents in the isoclinal positions

flexible conformations. Both the chair and boat conformations of cycloheptane exhibit torsional strain (Fig. 41b) [7].

Lower energy conformations are twist-chair and twistboat (Fig. 42). However, even twisting of the ring does not completely eliminate the strain. There is still some angle and torsional strain present in each conformation. The lowest energy conformation is a twist-chair, while a twistboat has slightly higher energy due to the presence of the intraannular "flagpole" hydrogen (Fig. 41b) [7]. Both the twist-chair and the twist-boat are in a state of pseudorotation similar to that of cyclopentane [19].

Conformational analysis of substituted cycloheptanes is rather complex. Each of the twist forms has seven different substituent positions and there are no "equatorial" or "axial" bonds. Each twist form, however, has one ring atom with two equivalent (isoclinal) substituent positions [20]. The lowest energy conformation of a geminally disubstituted cycloheptane has both substituents on that carbon (Fig. 43) [20]. 


\section{Conformational analysis of medium and large rings}

\section{Diamond lattice method for identifying low-energy conformations of cycloalkanes}

Diamond is an allotropic modification of carbon composed of $s p^{3}$-hybridized carbon atoms. In a diamond, carbon atoms assume arrangement described as a diamond lattice (Fig. 44). In a diamond lattice each carbon atom is bonded to four identical substituents-four other carbon atoms. Therefore, all the bond angles are ideal tetrahedral angles and all the bond lengths are $1.54 \AA$. Furthermore, all of the dihedral angles are either gauche $\left(60^{\circ}\right)$ or anti $\left(180^{\circ}\right)$. Thus, one can consider, to a first approximation, that any cyclic structure that can be superimposed to a diamond lattice is going to be a strain-free conformation [21]. In reality, that is the case with only a few rings. In cyclic compounds, each carbon atom is not bonded to four identical substituents and, hence, bond lengths and angles do not exactly correspond to the ideal tetrahedral values. Additional factors, such as transannular strain, which is not present in a diamond lattice, also play a role. However, diamond lattice method still has importance.

The diamond lattice method can be applied to sixmembered and larger rings as the smallest ring that can be superimposed on the diamond lattice is cyclohexane (Fig. 44b). Therefore, any smaller ring must exhibit angle strain. Furthermore, only rings with an even number of carbon atoms can form a closed path on a diamond lattice. As a result, all odd-membered rings must exhibit some torsional strain. We already encountered such situation with cycloheptane.

Even-membered rings can be classified into two types: those with $4 n$ and those with $4 n+2$ carbon atoms. Only rings with $4 n+2$ carbon atoms can have a completely strain-free conformation. An example is cyclohexane $(n=1)$. Cyclodecane $(n=2)$ is an exception. As a medium-sized ring, it exhibits steric (transannular) strain. Cyclotetradecane and larger $n+2$ rings are essentially strain free. Other rings with even number of carbon atoms $(4 n)$ exhibit some strain and for them the diamond lattice conformation is not always optimal. Among the large rings,
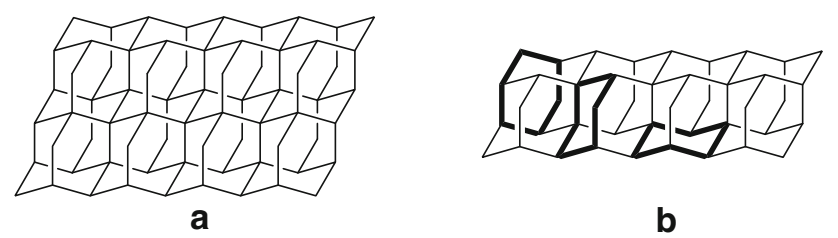

Fig. 44 a Diamond lattice. b Examples of six-membered rings superimposed on the diamond lattice those with odd number of carbon atoms exhibit the most strain.

While diamond lattice approach may represent a good starting point in conformational analysis of medium and large rings, nowadays its importance has decreased and we rely more on experimental (IR and Raman spectroscopies, $\mathrm{X}$-ray diffraction, electron diffraction, low-temperature ${ }^{1} \mathrm{H}$ and ${ }^{13} \mathrm{C}$ NMR) and theoretical approaches such as molecular mechanics (MM), molecular orbital (MO) and molecular dynamics (MD) methods.

\section{Medium rings}

\section{Common features of medium rings}

Compounds with $8-11$ atoms in a ring are classified as medium rings. Conformational analysis of medium rings is complex. They are not simply intermediate between common and large rings. They have some specific features that are characteristic only of them. Common and large rings generally differ little in chemical behavior compared to their open-chain counterparts.

Perhaps the most distinguishing feature of mediumsized rings is the difficulty in synthesizing them. Due to the both unfavorable enthalpy and entropy of activation ring closure reactions that result in formation of medium rings are difficult to carry out.

Furthermore, medium-sized rings are characterized by a relatively high strain. The strain per $\mathrm{CH}_{2}$ group in cyclooctane, cyclononane, cyclodecane and cycloundecane is rather high (Table 1; Fig. 45) [22]. Therefore, medium

Table 1 Ring strain of cycloalkanes

\begin{tabular}{lcc}
\hline Ring size & \multicolumn{2}{c}{ Strain per $\mathrm{CH}_{2}$} \\
\cline { 2 - 3 } & $\left.(\mathrm{kcal} \mathrm{mol})^{-1}\right)$ & $\left(\mathrm{kJ} \mathrm{mol}{ }^{-1}\right)$ \\
\hline 3 & 9.17 & 38.37 \\
4 & 6.58 & 27.53 \\
5 & 1.24 & 5.19 \\
6 & 0.02 & 0.08 \\
7 & 0.89 & 3.72 \\
8 & 1.21 & 5.06 \\
9 & 1.40 & 5.86 \\
10 & 1.24 & 5.19 \\
11 & 1.02 & 4.27 \\
12 & 0.34 & 1.42 \\
13 & 0.40 & 1.67 \\
14 & 0.14 & 0.59 \\
15 & 0.13 & 0.54 \\
16 & 0.12 & 0.50 \\
17 & -0.19 & -0.80 \\
\hline
\end{tabular}




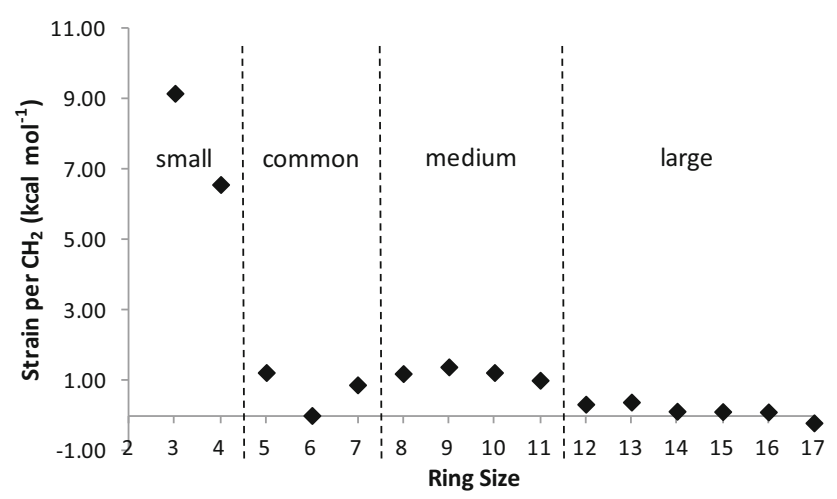

Fig. 45 Ring strain of cycloalkanes

rings always exhibit strain. In most cyclic compounds, the strain is a compromise between minimizing angle and torsional strain. In medium rings, angle and torsional strain are relatively low. However, they exhibit a unique type of steric strain. It is a consequence of conformations in which some of the bonds are directed into the ring. Such bonds and substituents are called intraannular bonds or substituents. Typically the substituent is a hydrogen atom since any substituent larger than hydrogen cannot occupy an intraannular position. Intraannular hydrogens "bump" into each other and exhibit steric hindrance called transannular strain. The substituents arranged outside the ring are called extraannular or peripheral substituents. Therefore, the overall strain is a result of a combination of bond angle distortion, partial eclipsing of hydrogens and transannular steric repulsions. It is not possible for medium-sized rings to relieve all of these strain-producing interactions in a single conformation. Thus, medium rings do not have a single low-energy conformation. Instead, there is an equilibrium among the several conformations that are close in energy to each other and are separated by low energy barriers. As ring gets larger, the strain decreases. There is a considerable reduction in strain in cyclododecane. This is an indication that it belongs to the category of large rings, which are characterized by low strain. Essentially strainfree conformations are attainable only for large-sized cycloalkanes, such as cyclotetradecane. In such rings, the carbon chain adopts a structure very similar to that of the straight-chain alkanes, having staggered hydrogens and an all-anti configuration. One can notice that the value for strain for cycloheptadecane is negative. Strain of a cycloalkane is not determined experimentally. Rather it is a comparison of experimentally determined values of heats of formation compared to hypothetical "unstrained" molecule [23].

Finally, there is a specific type of transannular reactions (reactions across the ring) that only medium-sized rings undergo. Such reactions are a consequence of conformations of medium rings. Their intraannular hydrogen substituents readily undergo 1,3- and 1,5-hydride shift resulting in rearranged products.

In summary, medium rings are characterized by three principal phenomena: low formation tendency, high strain and transannular reactions. Cycloheptane exhibits strain energy close to that of the medium rings. However, compared to eight- to eleven-membered rings, it is easy to prepare. Thus, it is classified as a common ring. On the other hand, cyclododecane, while difficult to prepare, has low strain energy. Therefore, it is a large ring.

Both from the names and from the actual conformations, one can infer that the elements of cyclohexane conformations are present in medium and larger rings. As the number of atoms in the ring increases, the number of conformations sharply increases. There is no formula or an algorithm to determine the number of conformations from the number of ring atoms.

While in small and common rings there was an attempt to identify and study all the conformations regardless of their energies, in medium and large rings an effort is made to clearly distinguish between a conformation, which is a local minimum on an energy diagram, and a transition state, which is a local maximum. While most efforts were placed in identification of all of the conformations of individual rings, study of transition states is also important. They represent pathways for interconversion of individual conformations, allowing one to identify the nature of the conformational "flip" (such as pseudorotation or inversion), the energies involved and hence how likely is that a particular conformation will be interconverted into another. Study of the transition states is also important when considering their reactivity and biomedical properties (such as interactions with enzymes and receptors).

\section{Cyclooctane}

Cyclooctane is the smallest member of the class of medium rings. It exhibits all of the typical properties of a medium ring: difficulty in cyclizing, transannular strain, transannular reactions and lack of single lowest energy structure. A total of 10 different conformations have been identified $[24,25]$. In fact, an additional conformation was reported [26], but it was apparently identical to the one already known [27]. Complexities of conformational analysis of cyclooctane are a very good illustration of the challenges encountered when dealing with medium and large rings.

On the diamond lattice there is a cyclic path involving eight carbon atoms (Fig. 46a). However, the resulting ring exhibits a high degree of strain. The two pairs of hydrogen atoms (indicated as $\mathrm{H}^{a}$ and $\mathrm{H}^{b}$ ) must point "inwards" (Fig. 46b), and are competing for the same positions in the diamond lattice, shown as red dots (Fig. 46c). Even though the carbon-hydrogen bond is shorter $(1.10 \AA)$ compared to 

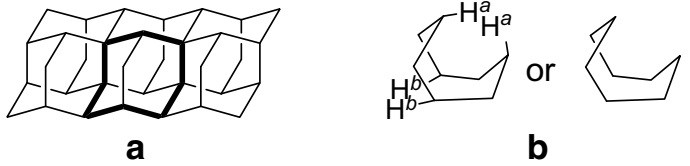

b
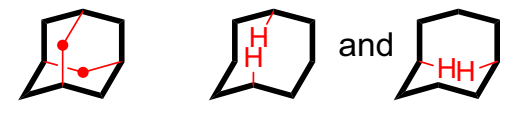

C
Fig. 46 a Conformation of cyclooctane obtained by following a closed path of eight carbon atoms on the diamond lattice. b Steric strain is a result of two pairs of hydrogen atoms competing for the



boat-boat

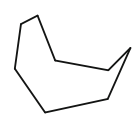

twist-boat
Fig. 47 Boat-boat and twist-boat are high energy conformations of cyclooctane

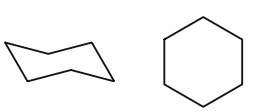

a

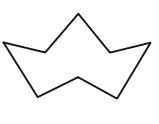

b

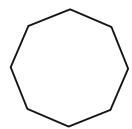

Fig. 48 Side-on perspective view (left) and view from the top (right) of a chair of cyclohexane, and $\mathbf{b}$ crown of cyclooctane

the carbon-carbon bond (1.54 $\AA$ ), still there is steric hindrance since the two hydrogen atoms should occupy the same space that a single carbon atom occupied in the diamond lattice. This is an example of transannular strain. Therefore, the conformation of cyclooctane derived from a diamond lattice, called boat-boat, is unstable. An alternative name for this conformation is "saddle". That is an unfortunate choice of a term, as there is a class of conformations that are saddle points on multidimensional energy diagrams. "Saddle" conformation of cycloctane is a specific conformation, better referred to as "boat-boat," and does not belong to the class of conformations that are saddle points.

The twist-boat conformation is obtained by a slight twist of the boat-boat to get interacting hydrogen atoms away from each other (Fig. 47). That relieves transannular strain, but increases torsional strain.

By analogy with the chair of cyclohexane, crown conformation of cyclooctane may appear to be of a low energy. However, symmetrical crown conformation has high torsional strain because of the high degree of eclipsing in all of the pseudoequatorial bonds (Fig. 48). Unlike the chair of cyclohexane, where neighboring equatorial bonds are gauche to each other, in the crown of cyclooctane they are eclipsing. Alternatively, one can slightly distort the crown conformation to obtain almost ideal torsion angles and bond angles. However, the resulting conformation has intraannular hydrogens on each side of the ring same positions (shown as red dots) in the lattice. c A more common representation of the same conformation

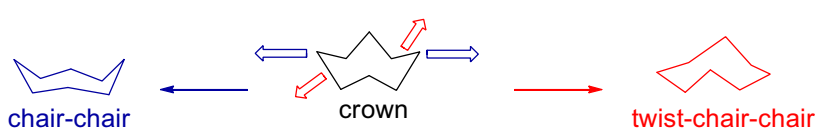

Fig. 49 Chair-chair and twist-chair-chair conformations of cyclooctane

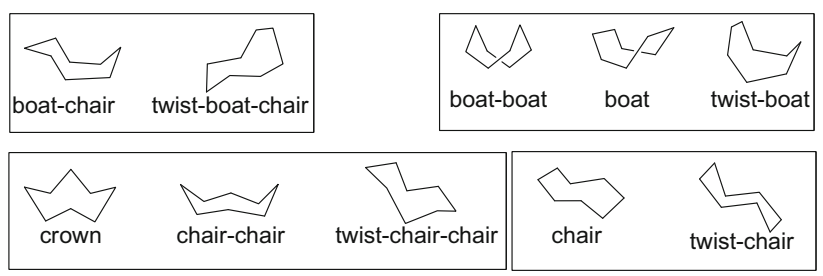

Fig. 50 There are four families of conformations of cyclooctane
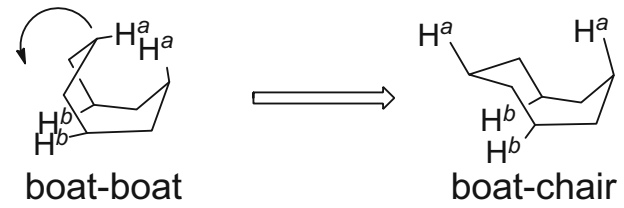

Fig. 51 Starting with diamond lattice-derived boat-boat conformation one can obtain the most stable conformation of cyclooctane

approaching very closely to each other resulting in a high steric strain.

Stretching the crown conformation between the opposite atoms results in a "chair-chair" conformation. Alternatively, stretching of the opposite bonds results in a "twistchair-chair". The latter is slightly favored (Fig. 49).

In summary, there are ten conformations of cyclooctane, which are divided into four families (Fig. 50) [24]. Interconversions within each family are by pseudorotation (a low energy barrier), while interconversions between different families are by inversion (a high energy barrier).

A hybrid between boat-boat and chair-chair conformations, the so-called boat-chair, has the lowest energy. Starting with diamond lattice-derived boat-boat conformation, the flip (Fig. 51), removes one of the transannular interactions directly as the two interacting hydrogens $\left(\mathrm{H}^{a}\right.$ pair) are moved away from each other. The second transannular interaction $\left(\mathrm{H}^{b}\right.$ pair) is also removed as the "flip" causes the remaining ring atoms to rotate and that 


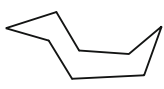

boat-chair $89 \%$

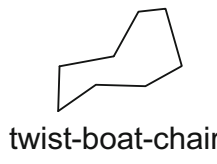

$7 \%$

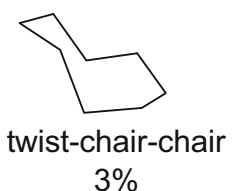

$3 \%$

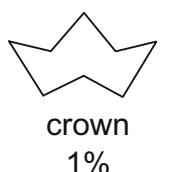

$1 \%$

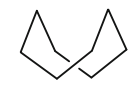

boat-boat

$0 \%$

Fig. 52 Conformations of cyclooctane with the amount of each present in the gas phase at $298 \mathrm{~K}$. The amounts were determined based on calculated free energies of the conformers [27]

Fig. 53 Conformations of cyclononane

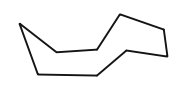

BC

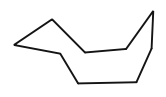

CB<smiles>ClC1CCCCCCCC1</smiles>

moves the second pair of interacting hydrogens away from each other. However, as a result of change of dihedral angles, the angle strain increases.

After the boat-chair, the next lowest energy conformation of cyclooctane is the twist-boat-chair followed by the twist-chair-chair. Crown and boat-boat are rather high in energy. At room temperature, boat-boat conformation is present only in trace amounts. Conformations such as boat or chair represent high-energy transition states. Therefore, as is the case with other medium rings, there is an equilibrium among the several conformations that are close in energy to each other and are separated by low energy barriers (Fig. 52) [27].

As a medium ring, cyclooctane also undergoes transannular reactions (reactions across the ring), which are covered later.

\section{Cyclononane}

Odd-membered medium and large rings have been studied somewhat less extensively compared to even-membered rings. Cyclononane has the highest strain of all medium ring cycloalkanes. Dale wrote that "On the basis of thermochemical strain cyclononane can be considered the most typical medium-sized ring. It can also be considered the smallest macrocyclic alkane inasmuch as torsion angles larger than $120^{\circ}$ start to play a role, and since the greater mobility of the ring permits conformational conversions to take place in more localized step processes rather than as synchronous changes of the whole ring" [28].

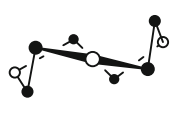

TBC [333]

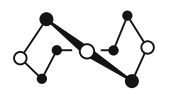

TCB [225]
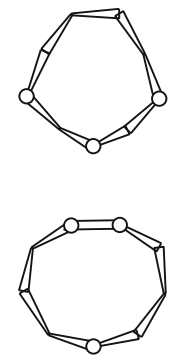

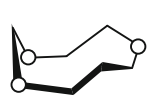

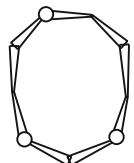

SCB [234]
Eight conformations that are cyclononane energy minima have been identified [29]. However, some conformations have relatively high energies and are separated from the lower energy conformations by extremely low energy barriers. Such conformations have relatively little thermodynamic significance. Cyclononane is the first case of a cycloalkane that does not have a lowest energy conformation. Rather it has several conformations of approximately equal energies that are separated by low energy barriers. Conformations of cyclononane are combinations of various chair and boat forms as named by Hendrickson [19, 30]. He identified boat-chair, chair-boat and chairchair forms as high-energy conformations [30]. Twisting of each gives the corresponding low-energy conformations of cyclononane (Fig. 53). Calculations show that a twistboat-chair (TBC) is the lowest energy form. It is a symmetric "triangular" conformation and is also designated as [333] conformation. Two other forms, twist-chair-boat (TCB) and twist-chair-chair (TCC), are relatively close in energy. Dale identified an additional, somewhat higher energy conformation as [234] or skew-chair-boat (SCB) [31]. Dale's representations and nomenclature is explained next. Corner carbon atoms are indicated as white circles and side atoms as black dots.

In the case of cyclononane the role of entropy is important. Usually when considering energy of a particular conformation, we consider only its enthalpy even though we should consider free energy. In most cases, particularly when determining only the relative stabilities of different conformations, the role of entropy is minor and can be 
Table 2 Variation in abundance of conformations of cyclononane with temperature

\begin{tabular}{llcc}
\hline Temperature & \multicolumn{2}{l}{ Abundance (\%) } \\
\cline { 2 - 4 } & TBC [333] & TCB [225] & TCC [144] \\
\hline$-173{ }^{\circ} \mathrm{C}$ & 95 & 5 & 0 \\
$\mathrm{rt}$ & 40 & 50 & 10 \\
\hline
\end{tabular}

neglected. That is not the case with cyclononane. The lowest energy conformation, TBC, has high symmetry and, thus, a low entropy. On the other hand a higher enthalpy conformation TCB also has considerably higher entropy as it is less symmetrical. As the contribution of entropy to the overall free energy increases with temperature $(\Delta G=\Delta H-T \Delta S)$, at the higher temperatures TCB becomes the major conformation. TCC conformation is undetectable by low-temperature ${ }^{13} \mathrm{C}$ NMR at $-173{ }^{\circ} \mathrm{C}$, barely detectable at $-95^{\circ} \mathrm{C}$ (estimated to be present in the amount of ca. $1 \%$ ) and present in significant amount at room temperature (Table 2) [32].

\section{Dale ("Wedge") representations of medium and large rings}

With cyclononane both the perspective drawings and the nomenclature of various conformations get somewhat confusing. Side-on perspective views suitable for representation of smaller rings make it difficult to visualize and analyze a larger ring conformation. There is also an issue with nomenclature. Naming is now less descriptive as it is rather difficult to draw conformation from a given name, or assign a unique unambiguous name to a conformation. Furthermore, there is considerable possibility for confusion as in the case of TCB, TBC and SCB.

Dale made an attempt to address these issues by introducing new conventions to represent and to name individual conformations [31]. In small and common rings each bond represents one "side" of the corresponding polygon (Fig. 54). In medium and large rings, bonds are aligned in such a way that two, or more bonds, are roughly linear and represent a side of a polygon. Dale represented the ring as viewed from the top and the individual bonds as wedges. Signs,+- (gauche) or $a$ (anti) are sometimes included to indicate conformation of the ring bonds. As mentioned earlier, hydrogen substituents in medium rings can be intraannular (pointing to the inside of the ring), or extraannular (pointing to the outside). Most carbon atoms in medium rings have one intraannular and one extraannular hydrogen. Such carbon atoms are on the "sides" of a polygon. A corner carbon atom has both extraannular hydrogen substituents (pointing "outward") and they are isoclinal.
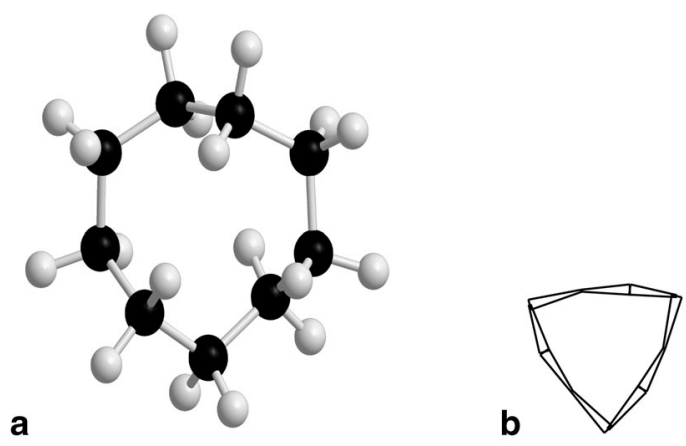

Fig. 54 Corner atoms in medium rings have both substituents pointing "outward," while side atoms have one pointing "inward" and the other one "outward". a Molecular model and b Dale's representation

There are numerous variations in Dale's representation of rings. In one form, open wedge bonds are used (Fig. 55a). They can be combined with filled circles to indicate corner atoms (Fig. 55b). Alternatively, all of the atoms can be indicated and one distinguishes side atoms from corner atoms by knowing that both carbon-carbon bonds on a corner atom must be gauche. That is sometimes a problem as in medium and large rings angles frequently enlarge to accommodate steric strain. That in turn, results in change in torsional angles and they frequently deviate from ideal gauche $\left(60^{\circ}\right)$ and anti $\left(180^{\circ}\right)$ angles. A more convenient definition of a corner atom is that it is the one with two extraannular isoclinal bonds. One can infer positions of hydrogens from the indicated carbon-carbon bonds. In a more complete representation, carbon-hydrogen bonds are indicated. Either filled (Fig. 55c) or open wedge bonds (Fig. 55d) can be used. Open circles are used to represent carbon atoms. $\mathrm{C}-\mathrm{H}$ bonds are indicated as wedges (above the plane) or solid lines (below the plane). While that provides a more complete picture of stereochemistry, sometimes the drawing is cluttered and extraannular bonds are not always clear.

From the indicated stereochemistry of ring carbons one can infer stereochemistry of the carbon-hydrogen bonds (Fig. 56). When there are two anti bonds on a carbon atom, it is a side carbon with one $\mathrm{C}-\mathrm{H}$ bond extraannular and the other one intraannular. Ring carbon atom with a gauche bond is a corner carbon with both $\mathrm{C}-\mathrm{H}$ bonds extraannular. From this representation it may not be clear that these bonds are also isoclinal. Note that, when carbon-hydrogen bonds are represented, ring carbons should be indicated with an open circle.

In most representations in this text hydrogen atoms have been omitted and open wedges are used to indicate carboncarbon bonds. Corner atoms are indicated as open circles (Fig. 57a). When needed a top-view perspective formula of the carbon skeleton, with hydrogen atoms omitted, was also included (Fig. 57b). 


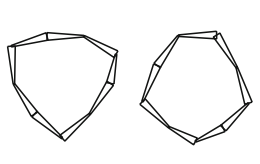

a

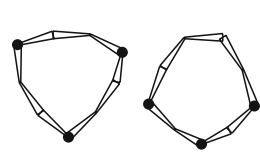

b

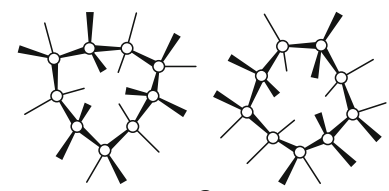

C

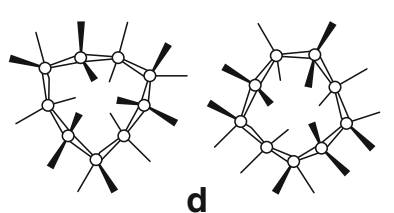

d

Fig. 55 Various forms of Dale's convention

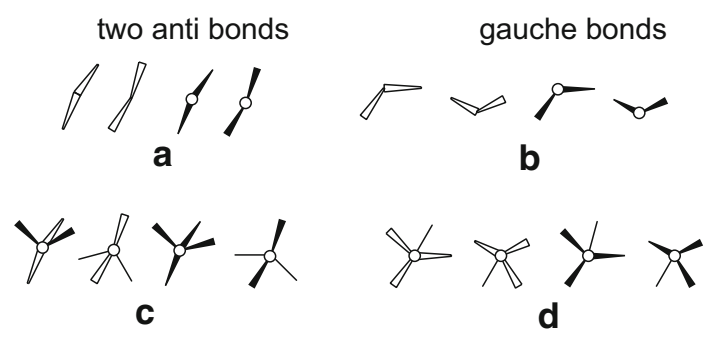

Fig. 56 Stereochemistry as indicated by Dale's convention: a anti bonds, $\mathbf{b}$ gauche bonds, $\mathbf{c}$ anti bonds with substituents, and $\mathbf{d}$ gauche bonds with substituents
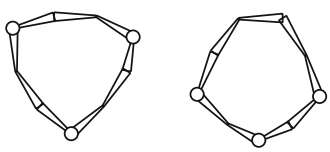

a

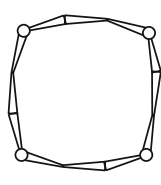

b



b
Fig. 57 Examples of representations used in this text: a [333] and [225] conformations of cyclononane, b [3333] conformation of cyclododecane represented as a Dale's formula (left) and a perspective drawing (right)

Each conformation is designated by a series of numbers within square brackets. Each number gives the number of bonds in one side, starting with the shortest and the direction of the ring is chosen so that the following number is the smallest possible [31]. For example, each "side" of TBC conformation has three bonds and the Dale notation is [333]. In SCB conformation the shortest side has two bonds, the shortest next to it three and the last one four. Hence, the notation is [234]. The sum of the numbers gives the ring size.

According to Dale convention, based on the number of sides, conformations of medium and large rings can be classified as triangular, quadrangular and quinquangular. All of the cyclononane conformations we examined are triangular. Quadrangular conformations are commonly encountered in even-membered large rings while quinquangular in odd-membered large rings.

\section{Cyclodecane}

The most studied and perhaps the most interesting medium-sized ring is that of cyclodecane. It is a very good illustration of the uniqueness of medium rings. For example, it is the only ring that has $4 n+2$ carbon atoms that exhibit significant strain.

Since cyclodecane has an even number of ring atoms, there is one closed diamond lattice path for the carbon skeleton. The result is a conformation designated as boatchair-boat (BCB) or [2323] (Fig. 58a). However, as was the case with cyclooctane, the two pairs of hydrogen atoms (pairs 1,5 and 6,10) are competing for the same positions (represented as red dots) in the diamond lattice. Thus, there is steric hindrance. To accommodate hydrogen atoms and reduce steric strain, bond angles become larger (on average $116^{\circ}$ ) and some bonds rotate out of the ideal staggered conformation. Therefore, steric strain is relieved at the expense of increased angle and torsional strain [33]. While that gets the two hydrogen atoms of each pair further apart, it also brings them closer to the third intraannular hydrogen on the same side of the ring (hydrogen 3 or 8 depending on the pair). As a result, on each side of the ring there are three hydrogen atoms cramped into a small space, which causes considerable steric (transannular) strain. In this conformation, a substituent larger than hydrogen cannot occupy an intraannular position. An alternative representation of the same conformation shows transannular interactions more clearly (Fig. 58b).

An alternative way to a diamond lattice approach to arrive at this conformation is to start with two fused cyclohexanes of trans-decalin in boat conformations (Fig. 59). Rotation of the two carbons "out" as indicated, causes change in bond and torsional angles resulting in the lowest energy, BCB, conformation.

Cyclodecane in its lowest energy conformation has six different types of hydrogen atoms and three types of carbon atoms. For comparison, in the chair conformation of cyclohexane there is only one type of carbon atom and two types of hydrogens (axial and equatorial). As the most stable structure is based on a diamond lattice, substituents can be classified as axial and equatorial. Definition of axial and equatorial bonds in rings other than cyclohexane was provided by Anet [34]. There are 6 axial and 14 equatorial hydrogens (Fig. 60). Also, there are 6 intraannular and 14 peripheral hydrogens. Intraannular hydrogens are of two types - two are axial (labeled as 1) and four are equatorial (substituents 2). Of the 14 peripheral hydrogen atoms, four are axial (substituents 3 ). The remaining 10 belong to three types: (1) four are attached to a carbon that contains a 
Fig. 58 Diamond lattice conformation and transannular strain in cyclodecane
Fig. 59 Derivation of the lowest energy conformation of cyclodecane starting with transdecalin
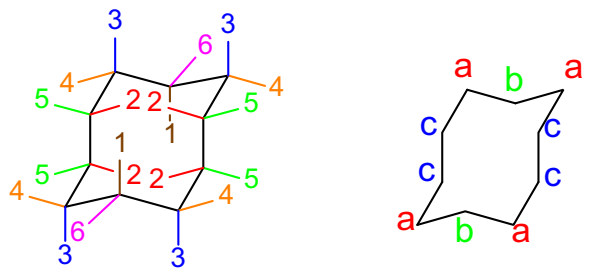

Fig. 60 Different types of hydrogen and carbon atoms in $\mathrm{BCB}$ conformation of cyclodecane

two $\mathrm{C}-\mathrm{H}$ bonds should fit here
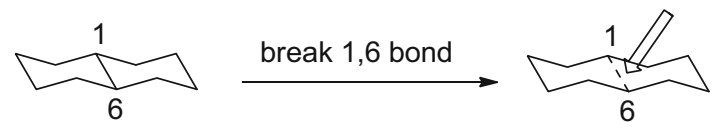

trans-decalin

Fig. 61 An alternative conformation of cyclodecane

peripheral axial hydrogen (substituents 4), (2) four are attached to the carbon that contains an intraannular equatorial hydrogen (substituents 5), and (3) two are attached to the carbon that contains an intraannular axial hydrogen (substituents 6). Therefore, four carbon atoms have both hydrogens in peripheral positions (a), two have one is peripheral and the other one is intraannular axial (b) and four have one is peripheral and the other one is intraannular equatorial (c).

There may be a temptation to represent cyclodecane as a trans-decalin (two fused cyclohexanes in chair conformations) with the "middle" bond (C1-C6) missing (Fig. 61). That is not a reasonable conformation as the steric hindrance between hydrogens 1 and 6 would be prohibitively
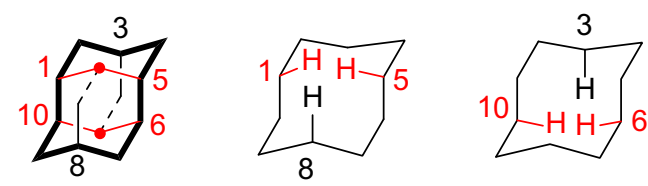

a
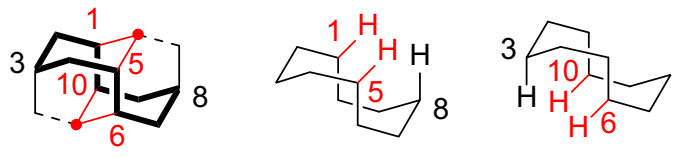

b

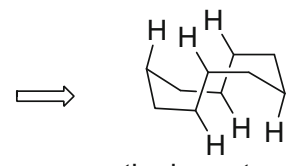

the lowest energy conformation of cyclodecane

large. As the C1-C6 bond of trans-decalin is broken in its place there would have to be a hydrogen atom on each carbon. However, the carbons 1 and 6 are separated by only $1.54 \AA$ and there is no space for hydrogen substituents. Obviously such structure is not reasonable. However, this conformation is a good starting point in search of a lowenergy one. As was the case with the diamond lattice-type conformation, by change in bond and torsional angles the two hydrogens are moved further apart. The resulting conformation, CCC (Fig. 62), is only slightly less stable compared to the diamond lattice-derived conformation.

There are 18 conformations of cyclodecane [29]. ${ }^{13} \mathrm{C}$ NMR studies have shown that at a low-temperature BCB is the principal conformer [35]. At room temperature, cyclodecane exists as a mixture of a rather large number of conformations. The six lowest energy conformations are shown in Fig. 62 [27, 36].

\section{Cycloundecane}

Cycloundecane is the least studied medium ring and only a few studies of its conformations are available. There are 41 conformations of cycloundecane identified by MM2 method and 32 identified by MM3 [29]. The most stable conformations of cycloundecane are either triangular or quinquangular [31]. ${ }^{13} \mathrm{C}$ NMR studies have shown that at a low temperature there is an equilibrium of two forms (Fig. 63) [37]. As the temperature increases other forms appear and, at a room temperature, cycloundecane is a mixture of several forms as indicated by its low melting point. Cycloundecane conformers cannot be described by the chair and boat nomenclature used for smaller rings and one has to use Dale nomenclature. 


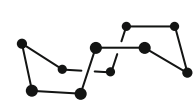

$\mathrm{BCB}$

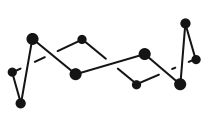

TBCC

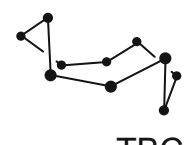

TBC
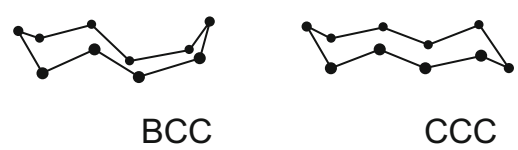

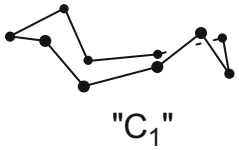

Fig. 62 The six lowest energy conformations of cyclodecane

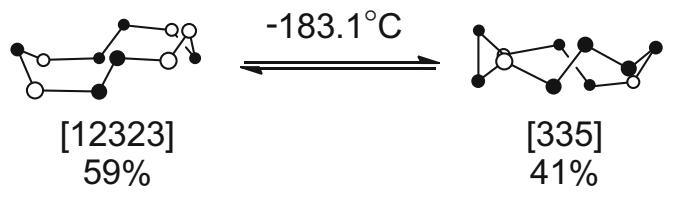

Fig. 63 Equilibrium of conformations of cycloundecane at a low temperature as determined by ${ }^{13} \mathrm{C}$ NMR

Since there is no closed path on the diamond lattice for an odd-membered ring, the diamond lattice method cannot be used directly. However, one still can start with the nearest even-membered ring and either add or truncate one carbon atom to arrive at the desired odd-membered ring [31]. Thus, in the case of cycloundecane one can start with the diamond lattice-derived [2323] conformation of cyclodecane and add one carbon atom (shown in red) to arrive at the cycloundecane conformation [12323] (Fig. 64a), or start with diamond lattice-derived conformation [3333] of cyclododecane and remove one carbon atom (shown in red) to arrive at the cycloundecane conformation [335] (Fig. 64b). Among the numerous other conformations of cycloundecane the four represented in Fig. 65 are closest in energy to the lowest energy [12323] and [335] conformations [37]. An eleven-member ring is the largest one that exhibits transannular reactions. Larger rings do not and that is one reason why rings larger than eleven-member are classified as large rings.

\section{Transannular reactions}

The most interesting features of medium-sized rings are the phenomena of transannular interactions and the

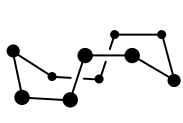

[2323]

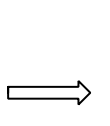

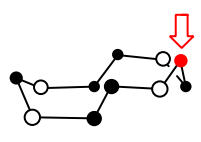

[12323]

a

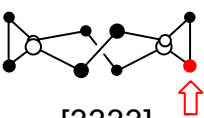

[3333] transannular reactions. Transannular interactions have been covered when dealing with each individual cycloalkane. In medium-sized rings, transannular reactions take place between atoms on opposite sides of the ring.

A transannular reaction is any reaction that occurs across a ring. All cyclic compounds, except of course a three-membered ring, can undergo transannular reactions. Thus, even cyclobutane undergoes a transannular reaction (Fig. 66) [38]. Transannular reactions on larger rings are common and are frequently exploited in synthesis. An example is the transannular aldol reaction (Fig. 67) [39]. Asymmetric transannular reactions have been recently reviewed [40].

There is a specific type of transannular reactions that occur only in medium rings [41]. Such reactions do not occur in open-chain compounds or other rings. Most of them involve 1,5-hydrogen shift. An example is bromination of cyclooctene with $\mathrm{N}$-bromoacetamide (NBA) in methanol (Fig. 68) [42]. Minor product, 1-bromo-2methoxycyclooctene, was the result of the expected 1,2addition to the double bond. Major products were the result of 1,5-hydride shift. Only in medium rings there is an intraannular hydrogen that is positioned for 1,5-hydride shift. Common rings underwent only the 1,2-addition reaction.

\section{Large rings}

Large rings are highly flexible. Properties of large rings resemble those of the corresponding open-chain compounds. As the rings get sufficiently large, they assume conformations of extended rectangles composed of two

Fig. 64 Generation of low energy conformations of cycloundecane starting with the diamond lattice-derived conformation of a cyclodecane and b cyclododecane

Fig. 65 Some additional low energy conformations of cycloundecane

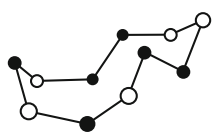

[13223]

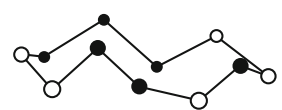

[12314]

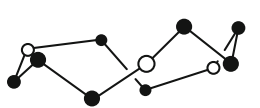

[344]

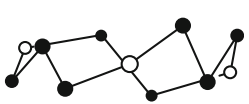

[344] 
long parallel chains linked by two bridges of minimum length [43]. Disubstituted derivatives do not exhibit cistrans isomerism since the rotation about carbon-carbon

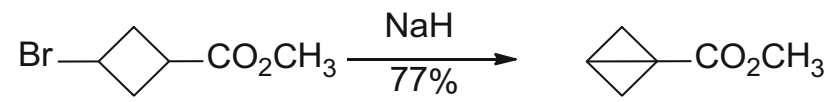

bonds in larger rings is free and the individual carbon atoms can rotate without affecting the rest of the ring. The number of conformations increases rapidly with the increasing ring size. For even-membered rings the number of possible diamond lattice conformations also increases and all large rings have more than one diamond lattice conformation.

Fig. 66 A transannular reaction of cyclobutane ester to give the corresponding bicyclo[1.1.0]butane ester

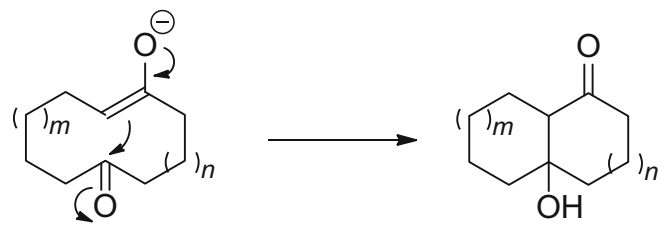

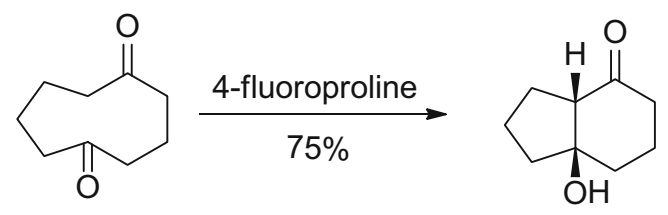

Fig. 67 Some examples of transannular reactions

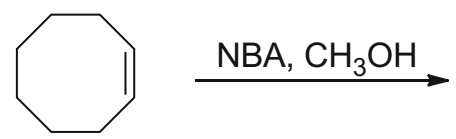<smiles>CO[C@H]1CCCCC[18O][C@@H]1Br</smiles><smiles>CO[C@H]1CC[C@@H](Br)CC[C@H]1C</smiles><smiles>BrC1CC=CCCCC1</smiles>

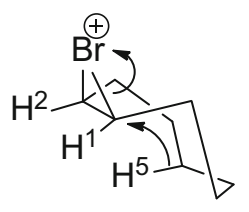

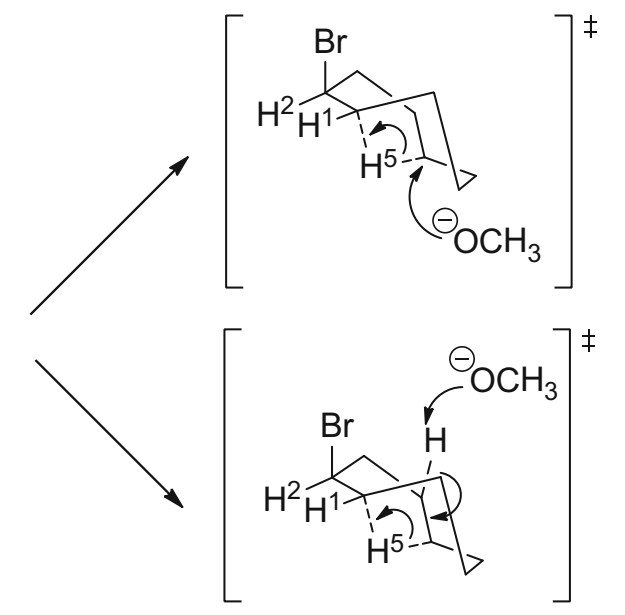<smiles>COC1CC2CC(OC)[C@H](C1)C2(Br)I</smiles>

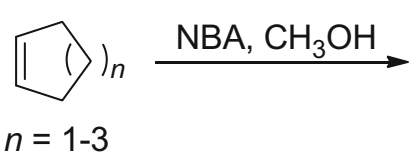<smiles>OC1CCCC1Br</smiles>

Fig. 68 Transannular reactions of cyclooctene 


\section{Cyclododecane}

Cyclododecane is considered to be the first large ring cycloalkane. It lends itself to some interesting line formulas (Fig. 69). Line formula a is almost never used as the size of the ring is not clear. Formula $b$ is the one that is most commonly used. It clearly indicates the number of carbon atoms in the ring and it can represent both cis and trans double bonds in a ring. Formulas $\mathrm{c}$ and $\mathrm{d}$ are sometimes encountered.

Weinberg and Saul found 97 conformations of cyclododecane utilizing MM3 and 121 utilizing MM2 method [29]. Even though there is a large number of different conformations, there is an agreement that cyclododecane exists as a single low-energy conformation, designated as [3333] (Fig. 70), with other conformations being considerably higher in energy. That is supported by both experimental results [44] and theoretical calculations $[18,45]$. Thus, considering cycloalkanes in the order of increasing ring size, this is the first case since cyclohexane that a single low-energy conformation can be assigned to a cycloalkane.

The lowest energy conformation of cyclododecane is not of diamond lattice type. There are four diamond lattice
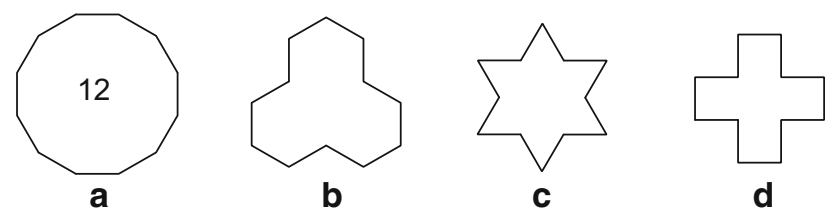

Fig. 69 Various line formulas of cyclododecane
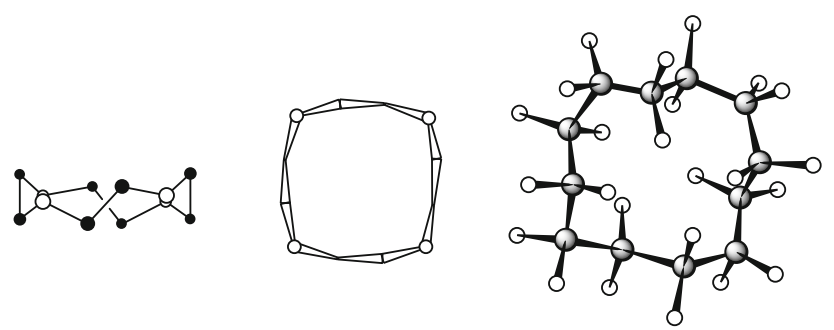

Fig. 70 [3333] conformation of cyclododecane

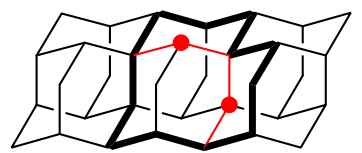

a

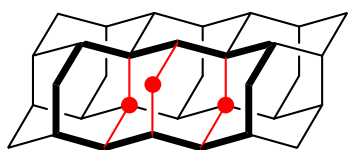

b
Fig. 71 The diamond-lattice conformations of cyclododecane: a the conformation that exhibits two pairs of hydrogen-hydrogen interactions, b the conformation that exhibits three pairs of hydrogen- conformations (Fig. 71) [21]. All of the diamond latticetype conformations have "overlap" interactions (interactions where hydrogen substituents compete for the same position in the diamond lattice indicated as a red dot), which renders them high in energy. For example, conformation a has two hydrogen-hydrogen interactions, conformation $\mathrm{b}$ three, while each of the conformations $\mathrm{c}$ and $\mathrm{d}$ has one serious "triple hydrogen" interaction.

\section{Cyclotridecane}

The conformational analysis of cyclotridecane is complex, as is common for odd-membered rings. Only a few theoretical and experimental studies were carried out so far and relatively little is known. Low-temperature NMR studies have been one of the best methods to study conformational equilibria of cycloalkanes. Cyclotridecane undergoes a fast pseudorotation and low-temperature NMR studies did not yield any useful results [46]. According to the strain energy calculations, there are five low-energy conformations, which are separated by low energy barriers (Fig. 72) [31, 47]. Two quinquangular conformations are of the lower energy, while three triangular are of somewhat higher. Based on the calculations [48] and the studies of related systems [46, 49], some authors have proposed conformation [12433] [29, 31, 48] while others [13333] [46, 47] to be the lowest energy conformation.

Recently a [337] was proposed as the lowest energy conformation [49]. It is a variation of [13333] conformation with corner atoms 2 and 3 now being now a part of one long seven-carbon side (Fig. 73). One should note that the study was based on a ring in which carbon 1 was $s p^{2}$ hybridized. Thus, a distinction between a triangular [337] and quinquangular [13333] conformation may not be clear. About 35 years earlier, Dale himself predicted such a possibility: "Sometimes there is even a problem of classification, since a definition based on whether the ring projection "looks" triangular or quinquangular, and a definition based on whether the relevant dihedral angles are above or below $120^{\circ}$, may lead to different results" [31]. However, it should be noted that in the same paper Dale identified [337] as a high-energy conformation of cyclotridecane.

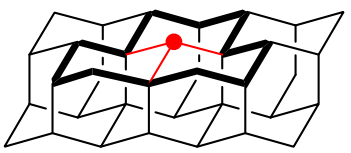

C

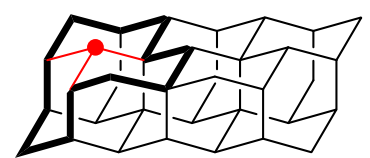

d hydrogen interactions, and c, d two conformations that exhibit a "triple hydrogen" interaction 
Fig. 72 Low energy conformations of cyclotridecane

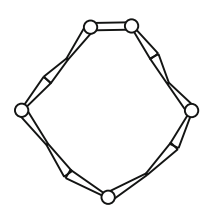

[13333]

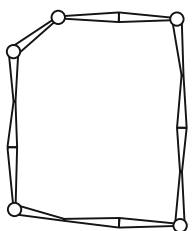

[12433]

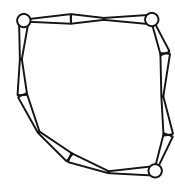

[346]

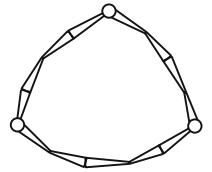

[445]

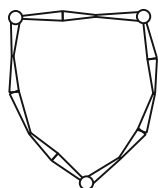

[355]
Fig. 73 a [13333] and [337] conformations of cyclotridecane with hydrogens omitted, corner carbon atoms are shown in blue and the $s p^{2}$-hybridized carbon atom is shown in green. $\mathbf{b}$ [337] $\mathrm{X}$-ray structure of 2,4dinitrophenylhydrazone of cyclotridecanone [49] conformation is based on the

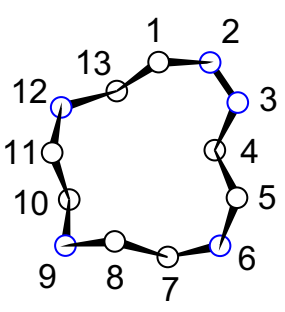

[13333]

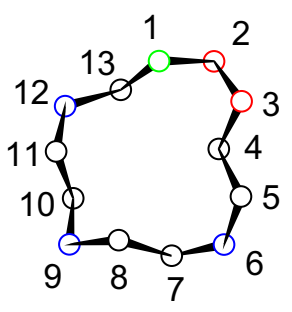

[337] a

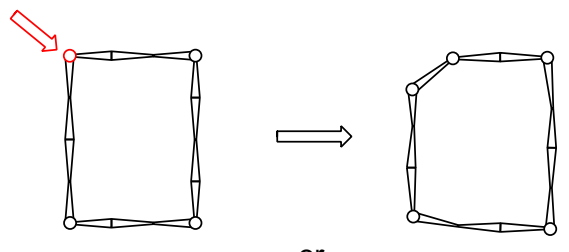

or

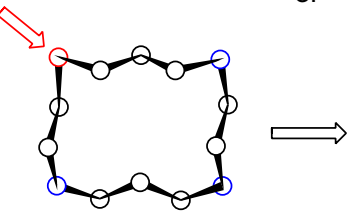

[3434]<smiles>C1OOOOOOOOOOO1</smiles>

[12433]

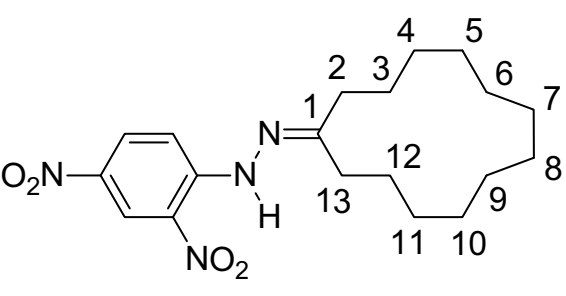

b energy conformations of diamond lattice-derived conformation of b cyclododecane. Carbon atom indicated by an arrow and shown in red is a removed or b added
Fig. 74 Generation of low

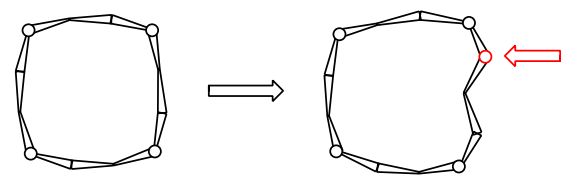

or<smiles>C=COOOOCOOOOOOOOOOOOOOOOOOOOOOOO</smiles>

[3333]
[13333] b
As in the case of cycloundecane, two lowest energy conformations can be derived from the diamond lattice conformations of the nearest even-membered rings (Fig. 74). Conformation [12433] may be derived by ring contraction from the diamond lattice conformation [3434] of cyclotetradecane. Conformation [13333] can be derived from the [3333] conformation of cyclododecane by ring expansion.

\section{Cyclotetradecane}

Cyclotetradecane is the smallest saturated ring (in addition to cyclohexane) that is strain free. It has a diamond latticetype conformation [3434] (Fig. 75a), with enough space for all interior hydrogen atoms (Fig. 75b,c) [31]. The substituents, however, can only occupy exterior positions, and geminal substituents only the corner positions. A non- diamond-lattice conformation [3344] (Fig. 75d) is the nextbest candidate and has been calculated to be about $2.6 \mathrm{kcal} \mathrm{mol}^{-1}$ higher in energy.

\section{Cyclopentadecane}

There are five low-energy conformations of cyclopentadecane according to the strain energy calculations [31, 47]. All of the low-energy conformations are quinquangular (Fig. 76). The highly symmetrical [33333] conformation has the lowest strain energy. However, it also has low entropy. When entropy is taken into account, at room temperature, conformations [13443] and [14334] have about the same free energy as [33333] and the remaining two, [13434] and [13353], only slightly higher. Therefore, at room temperature cyclopentadecane is a mixture of the five low-energy conformations shown in Fig. 76. 


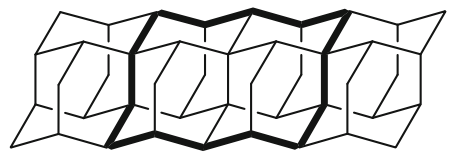

a

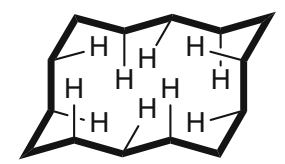

b
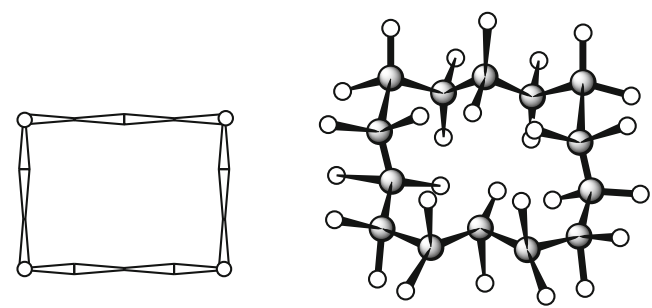

C
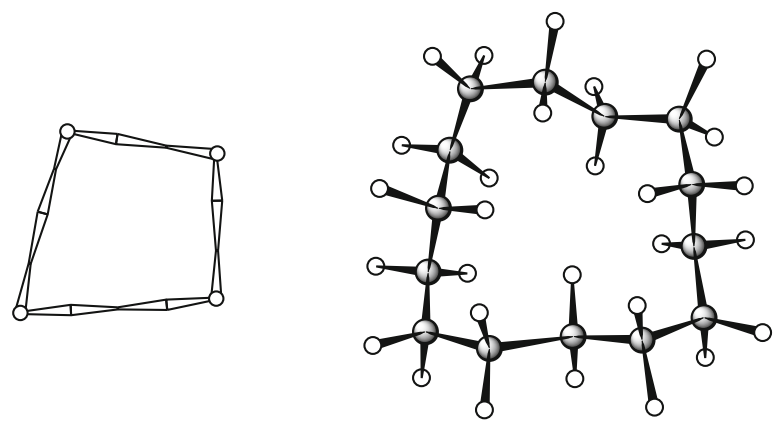

d

Fig. 75 a The lowest energy [3434] conformation of cyclotetradecane generated from the diamond lattice. b [3434] Conformation showing internal hydrogen atoms. c [3434] conformation. d [3344] conformation

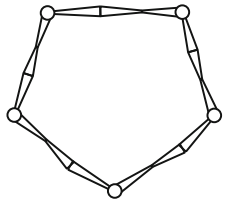

[33333]

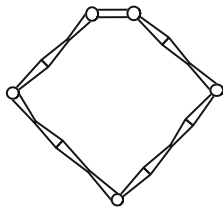

[13443]

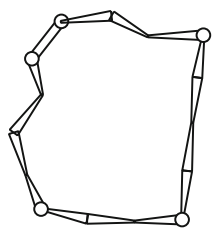

[13434]

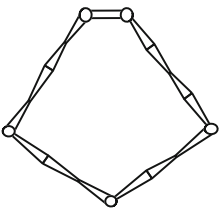

[14334]

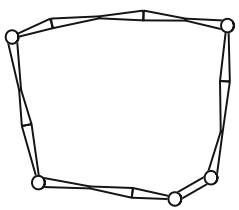

[13353]

Fig. 76 Low energy conformations of cyclopentadecane

\section{Larger rings}

As expected, complexity of conformational situation increases with the ring size. Conformational analysis becomes more complex and there are fewer studies available. However, one can still draw some general conclusions. With the increase in ring size the number of possible conformations sharply increases and finding all reasonable conformations becomes a problem [29]. Larger rings are likely to exist as mixtures of several conformations. Evenmembered rings exhibit low strain and tend to be mixtures of various quadrangular conformations. Odd-membered rings are more strained and are usually more complex mixtures of a larger number of various, mainly quadrangular and quinquangular, conformations.

The most stable conformation of cyclohexadecane is the diamond lattice [4444] conformation [31] (Fig. 77a). It is $1.9 \mathrm{kcal} \mathrm{mol}^{-1}$ more stable compared to the next most stable conformation [3535] [50]. More recent molecular dynamics structures indicate that, at higher temperatures, cyclohexadecane is a mixture of a large number of different conformations with [4444] still being the most abundant, but the second most populated conformer is [3445] [51].

Cycloheptadecane presents an interesting problem for conformational analysis. Since it is a large odd-membered ring, there is no obvious low-energy conformation. Cycloheptadecane is a challenging target and search for all of its conformations has been used as a test for validity of a particular search method $[29,52,53]$. There is an extremely large number of conformational minima and 264 conformations in the range of $0-3 \mathrm{kcal} \mathrm{mol}^{-1}$ have been identified [52]. Conformation shown in Fig. 77b has been identified as the preferred conformation of cycloheptadecane $[53,54]$.

Conformations of even larger rings become even more interesting as the increased conformational flexibility and attractive van der Waals interaction begin to play a role. With the increase in ring size long and thin rectangular 


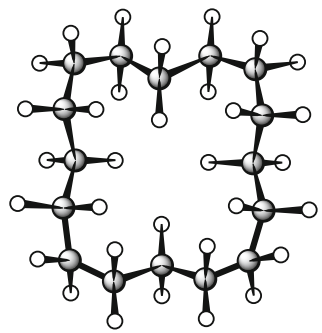

[4444]

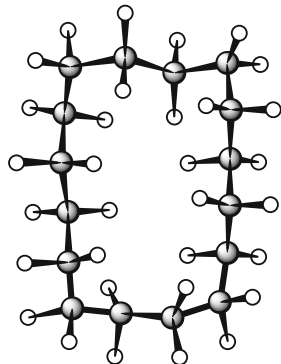

[3535]

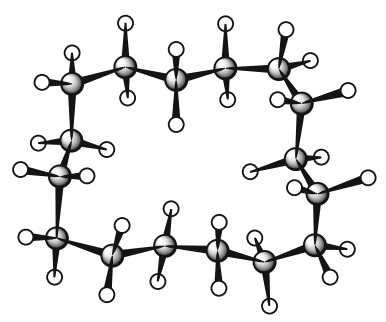

[3445]

a

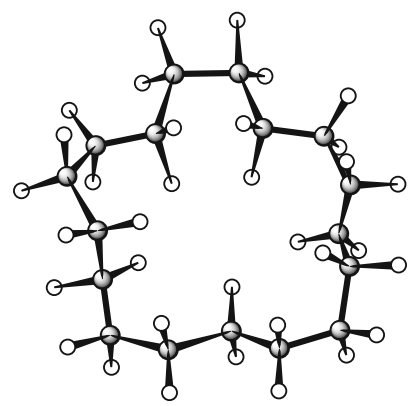

b

Fig. 77 Conformations of a cyclohexadecane and $\mathbf{b}$ cycloheptadecane

Fig. 78 Conformations of cyclooctadecane in the order of increasing strain energy a [3636], b [4545], and c [234234]
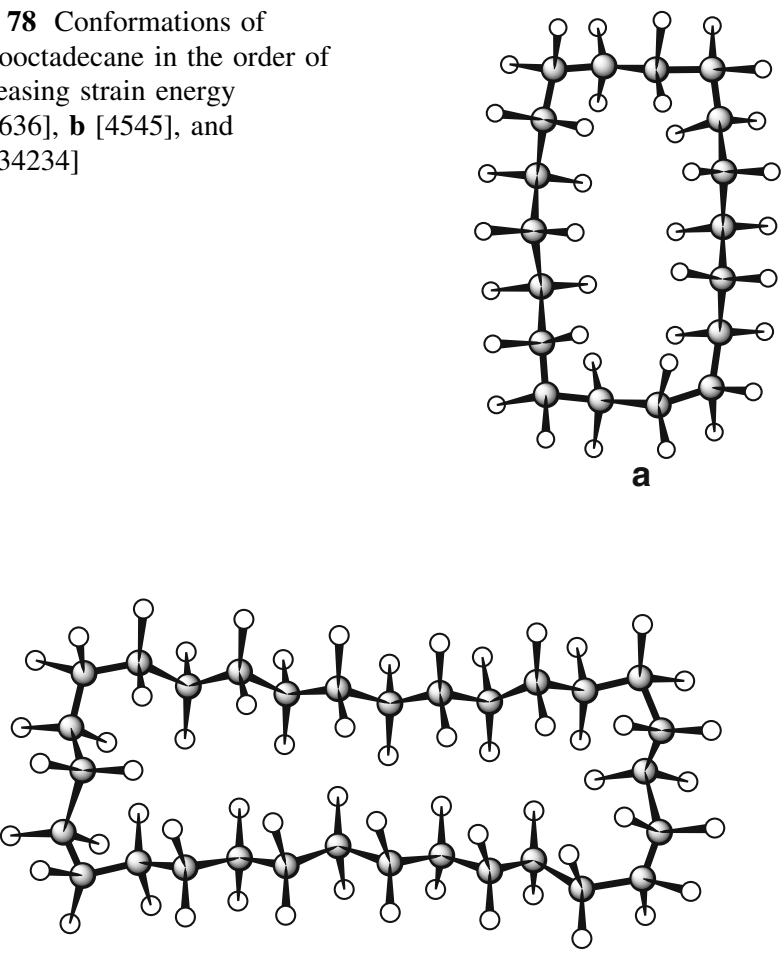

Fig. 79 In cyclotriacontane $\left(\mathrm{C}_{30} \mathrm{H}_{60}\right)$ the attractive van der Waals interactions cause the ring to be distorted inwards

conformations become preferred over the square ones. The lowest energy conformation of cyclooctadecane is [3636] while [4545] has a slightly higher energy (Fig. 78) [55]. While in medium rings, molecules underwent valence angle and torsion angle distortion to move the intraannular hydrogens further apart, large rings, such as cyclooctadecane, assume conformations that bring them closer together and maximize attractive van der Waals interactions (a more accurate though less commonly used term is London forces). In the [4545] conformer intraannular hydrogens are further apart resulting in a decreased van der Waals stabilization. By flipping inwards two carbons on the opposite


"long" sides of the conformation [4545] one obtains the next most stable conformation, which can be designated as [234234] (Fig. 78c). Note that in it the intraannular hydrogens are closer to other intraannular hydrogens compared to [4545] conformation. Cyclooctadecane is the smallest ring in which such conformation is possible without causing transannular strain. In cyclooctacosane $\left(\mathrm{C}_{28} \mathrm{H}_{56}\right)$ and larger cycloalkanes the attractive van der Waals forces can actually cause the ring to be distorted inwards (Fig. 79) [55].

The lowest energy conformations of cyclononadecane and cycloicosane are analogous to those of cyclooctadecane (Fig. 80).

\section{Conclusion}

Conformational analysis is an indispensible tool for elucidation of the properties and behavior of organic molecules. Molecules should not be considered to be static "frozen" species as implied by molecular models. To fully understand three-dimensional structure of molecules, one has to consider their flexibility, thermal motion and a possibility of a bond rotation. Furthermore, conformational analysis 

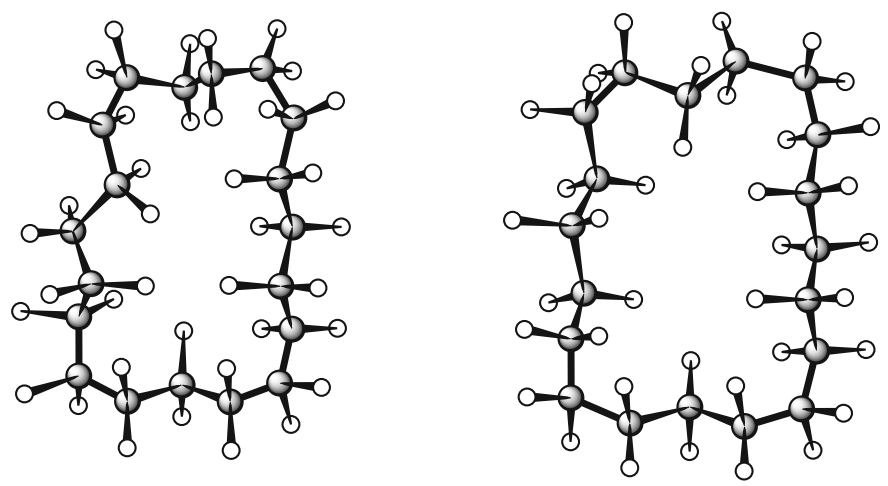

a

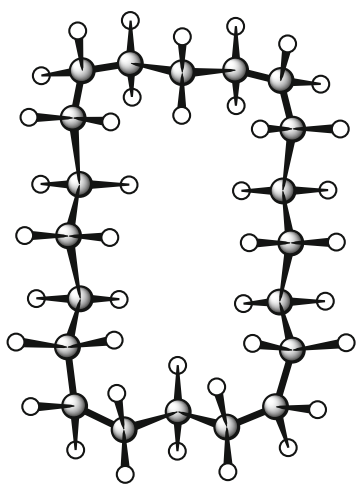

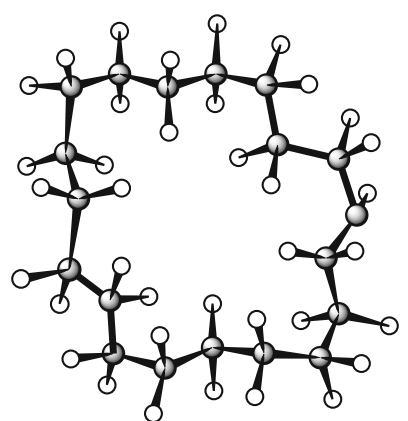
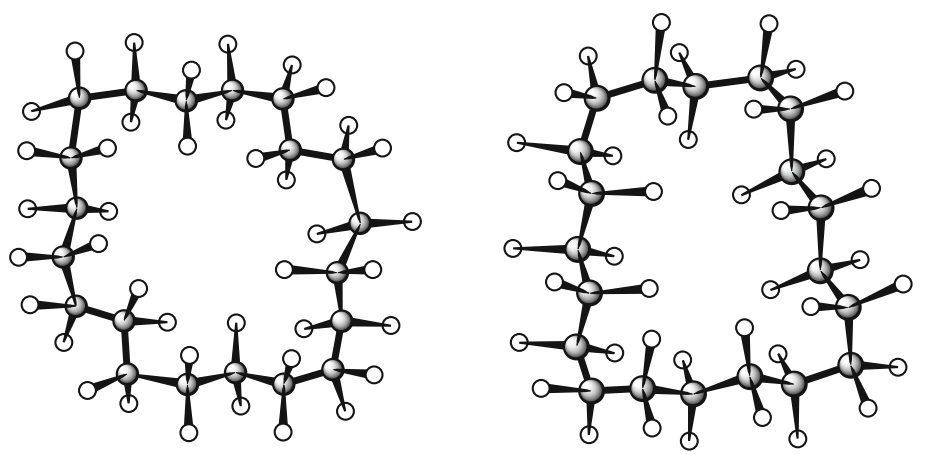

b

Fig. 80 The lowest energy conformations in the order of increasing strain energy of a cyclononadecane and $\mathbf{b}$ cycloicosane

should be related to chemical thermodynamics and in particular theory of intermediates and transition states. Finally, in the case of medium and large rings, the role of entropy cannot be neglected.

In a typical organic chemistry course, conformational analysis of cycloalkanes is restricted to small and common rings. Medium and large rings are present in numerous natural products and pharmacologically active compounds. Thus, not only a thorough understanding, but also an ability to apply conformational analysis is essential to comprehend biological properties of organic compounds and interactions in complex systems.

\section{References}

1. Van 't Hoff JH (1874) Archives neerlandaises des sciences exactes et naturelles 9: 445-454

2. Le Bel JA (1874) Bull Soc Chim France 22:337-347

3. Barton DHR (1950) Experientia 6:316-320

4. Newman MS (1955) J Chem Educ 32:344-347

5. Pophristic V, Goodman L (2001) Nature 411:565-568

6. Basic Terminology of Stereochemistry (IUPAC Recommendations 1996) http://www.chem.qmul.ac.uk/iupac/stereo/TZ.html\#05. Accessed 29 June 2015

7. Hendrickson JB (1961) J Am Chem Soc 83:4537-4547
8. Allinger NL (2011) J Comput Aided Mol Des 25:295-316

9. Ramsay O (1977) J Chem Educ 54:563

10. Dragojlovic V (2001) J Chem Educ 78:923

11. Leventis N, Hanna SB, Sotiriou-Leventis C (1997) J Chem Educ 74:813-814

12. Nelson DJ, Brammer CN (2011) J Chem Educ 88:292-294

13. Pauling L (1931) J Am Chem Soc 53:1367-1400

14. Bernett WA (1967) J Chem Educ 44:17-24

15. Burger DJ (1998) Walsh Cyclopropane Molecular Orbitals http:// www.bluffton.edu/ bergerd/chem/walsh/cp.html. Accessed 29 June 2015

16. Dale J (1978) Stereochemistry and Conformational Analysis. Verlag Chemie, New York, Weinheim, p 141

17. Pitzer KS, Dona WT (1959) J Am Chem Soc 81:3213-3218

18. Kolossvary I, Guida WC (1993) J Am Chem Soc 115:2107-2119

19. Hendrickson JB (1967) J Am Chem Soc 89:7047-7061

20. Hendrickson JB, Boeckman RK Jr, Glickson JD, Grunwald E (1973) J Am Chem Soc 95:494-505

21. Saunders M (1967) Tetrahedron 23:2105-2113

22. Eliel EL, Wilen SH (1994) Stereochemistry of organic compounds. Wiley, New York, pp 675-678

23. Wiberg KB (1986) Angew Chem Int Ed Engl 25:312-322

24. Hendrickson JB (1967) J Am Chem Soc 89:7036-7043

25. Anet FAL, Krane J (1973) Tetrahedron Lett 5029-5032

26. Rocha WR, Pliego JR Jr, Resende SM, Dos Santos HF, De Oliveira MA, De Almeida WB (1998) J Comput Chem 19:524-534

27. Wiberg KB (2003) J Org Chem 68:9322-9329

28. Dale J (1978) Stereochemistry and Conformational Analysis. Verlag Chemie, New York, Weinheim, p 204

29. Weinberg N, Wolfe S (1994) J Am Chem Soc 116:9860-9868

30. Hendrickson JB (1964) J Am Chem Soc 86:4854-4866 
31. Dale J (1973) Acta Chem Scand 27:1115-1129

32. Anet FAL, Krane J (1980) Isr J Chem 20:72-83

33. Dale J (1978) Stereochemistry and Conformational Analysis. Verlag Chemie, New York, Weinheim, pp 207-208

34. Anet FAL (1990) Tetrahedron Lett 31:2125-2126

35. Pawar DM, Smith SV, Mark HL, Odom RM, Noe EA (1998) J Am Chem Soc 120:10715-10720

36. Pawar DM, Cain D, Gill G, Bain AD, Sullivan RH, Noe EA (2007) J Org Chem 72:25-29

37. Pawar DM, Brown J II, Chen K-H, Allinger NL, Noe EA (2006) J Org Chem 71:6512-6515

38. Wiberg KB, Lampman GM, Ciula RP, Connor DS, Schertler P, Lavanish J (1965) Tetrahedron 21:2749-2769

39. Chandler CL, List B (2008) J Am Chem Soc 130:6737-6739

40. Reyes E, Uria U, Carrillo L, Vicario JL (2014) Tetrahedron 70:9461-9484

41. Cope AC, Martin MM, McKervey MA (1966) Q Rev Chem Soc 20:119-152

42. Fresnet P, Dubois J-E (1979) Tetrahedron Lett 2137-2140

43. Dale J (1963) J Chem Soc 93-111

44. Atavin EG, Mastryukov VS, Allinger NL, Almenningen A, Seip R (1989) J Mol Struct 212:87-95 (and references cited therein)
45. Yates PC, Richardson CM (1996) J Mol Struct (Theochem) 363:17-22

46. Rubin BH, Willianson M, Takeshita M, Menger FM, Anet FAL, Bacon B, Allinger NL (1984) J Am Chem Soc 106:2088-2092

47. Anet FAL, Rawdah TN (1978) J Am Chem Soc 100:7810-7814

48. Dos Santos HF, Franco ML, Venancio MF, Ferreira DEC, Anconi CPA, Rocha WR, De Almeida WB (2012) Int J Quantum Chem 112:3188-3197

49. Valente EJ, Diwaker MP, Fronczek FR, Noe EA (2008) Acta Cryst. C64:0447-0449

50. Anet FAL, Cheng AK (1975) J Am Chem Soc 97:2420-2424

51. Zhang R, Mattice WL (1993) J Chem Phys 98:9888-9894

52. Kolossvary I, Guida WC (1999) J Comp Chem 20:1671-1684

53. Saunders M, Houk KN, Wu Y-D, Still WC, Lipton M, Chang G, Guida WC (1990) J Am Chem Soc 112:1419-1427

54. Stephen M, Long SM, Tran TT, Adams P, Darwen P, Smythe ML (2011) J Comput Chem 32:1541-1549

55. Shah AV, Dolata DP (1993) J Comput Aided Mol Des 7:103-124 